\title{
Low Frequency and Weighted Likelihood Solutions for Mixed Frequency Dynamic Factor Models
}

Francisco Blasques*

Siem Jan Koopman*

Max Mallee

* Tinbergen Institute 
Tinbergen Institute is the graduate school and research institute in economics of Erasmus University Rotterdam, the University of Amsterdam and VU University Amsterdam.

More TI discussion papers can be downloaded at http://www.tinbergen.nl

Tinbergen Institute has two locations:

Tinbergen Institute Amsterdam

Gustav Mahlerplein 117

1082 MS Amsterdam

The Netherlands

Tel.: +31(0)205251600

Tinbergen Institute Rotterdam

Burg. Oudlaan 50

3062 PA Rotterdam

The Netherlands

Tel.: +31(0)10 4088900

Fax: $+31(0) 104089031$

Duisenberg school of finance is a collaboration of the Dutch financial sector and universities, with the ambition to support innovative research and offer top quality academic education in core areas of finance.

DSF research papers can be downloaded at: http://www.dsf.nl/

Duisenberg school of finance

Gustav Mahlerplein 117

1082 MS Amsterdam

The Netherlands

Tel.: +31(0)20 5258579 


\title{
Low Frequency and Weighted Likelihood Solutions for Mixed Frequency Dynamic Factor Models
}

\author{
Francisco Blasques, Siem Jan Koopman and Max Mallee* \\ VU University Amsterdam, Department of Econometrics \\ Tinbergen Institute Amsterdam
}

August 10, 2014

\begin{abstract}
The multivariate analysis of a panel of economic and financial time series with mixed frequencies is a challenging problem. The standard solution is to analyze the mix of monthly and quarterly time series jointly by means of a multivariate dynamic model with a monthly time index: artificial missing values are inserted for the intermediate months of the quarterly time series. In this paper we explore an alternative solution for a class of dynamic factor models that is specified by means of a low frequency quarterly time index. We show that there is no need to introduce artificial missing values while the high frequency (monthly) information is preserved and can still be analyzed. We also provide evidence that the analysis based on a low frequency specification can be carried out in a computationally more efficient way. A comparison study with existing mixed frequency procedures is presented and discussed. Furthermore, we modify the method of maximum likelihood in the context of a dynamic factor model. We introduce variable-specific weights in the likelihood function to let some variable equations be of more importance during the estimation process. We derive the asymptotic properties of the weighted maximum likelihood estimator and we show that the estimator is consistent and asymptotically normal. We also verify the weighted estimation method in a Monte Carlo study to investigate the effect of different choices for the weights in different scenarios. Finally, we empirically illustrate the new developments for the extraction of a coincident economic indicator from a small panel of mixed frequency economic time series.
\end{abstract}

Keywords: Asymptotic theory, Forecasting, Kalman filter, Nowcasting, State space. JEL classification: C13, C32, C53, E17.

\section{INTRODUCTION}

The multivariate analysis of a panel of economic and financial time series with mixed frequencies can be treated by a range of different approaches. The problem of mixed frequency time series is regarded as a challenging problem in many applied econometric

\footnotetext{
*Contact: f.blasques@vu.nl, s.j.koopman@vu.nl and m.i.p.mallee@vu.nl
} 
studies. Currently there are two main competing approaches in the literature to handle mixed frequency time series: partial model and full system methods. This classification is adopted from Banbura, Giannone, Modugno, and Reichlin (2013). For the partial model solution, the multivariate model specifications focus particularly on low frequency time series variables while high frequency explanatory variables are aggregated to the lower frequency. The dynamics of the explanatory variables are not described by the model. When the full system method is adopted, the low and high frequency variables are modeled simultaneously. This approach can model feedback between variables since all variables are endogenous. In most full system methods all variables are modeled at the high frequency where series observed at a low frequency have missing values.

The most commonly used partial model methods are Bridge models and Mixed Data Sampling (MIDAS) models. In Bridge models the high frequency data are forecasted up to the desired forecast horizon in a separate time series model. These forecasts are then aggregated to the lower frequency and are used as explanatory variables in a lower frequency time series model as contemporaneous values. Bridge models are often used to forecast quarterly Gross Domestic Product (GDP) using a set of monthly observed indicators. Trehan (1989) is the first application of Bridge equations in this setting. Baffigi, Golinelli, and Parigi (2004) and Golinelli and Parigi (2007) use Bridge models where GDP is predicted by the National Accounts income-expenditure identity. The MIDAS approach was proposed by Ghysels, Santa-Clara, and Valkanov (2006). As in Bridge models, the series with the lower frequency are regressed on the series with the higher frequency. However, when forecasting with MIDAS regression only one step is required. To obtain forecasts at horizon $h$, the values of $y_{t}$ are simply regressed on the values of the indicators up to period $t-h$ and the dynamics of the regressors are not specified by the model. In MIDAS regressions, the higher frequency series are not aggregated, but each lag has its own regression coefficient. To avoid parameter proliferation, the coefficients of the different lags are described by a weighting function. Foroni, Marcellino, and Schumacher (2012) propose the use of unconstrained distributed lags of the high frequency indicators. This approach is referred to as unrestricted MIDAS or U-MIDAS.

Marcellino, Carriero, and Clark (2014) propose a method for producing currentquarter forecasts of GDP growth with a large range of available within-the-quarter monthly observations of economic indicators. Each time series of monthly indicators is transformed into three quarterly time series, each containing observations for, respectively, the first, second or third month of the quarter. Hence, there can be missing observations at the end of some of these three time series, depending on the specific month of the quarter. They include in the model only the constructed quarterly series without missing observations at the moment in time the forecast is formed. They use Bayesian methods to estimate the resulting model, which expands in size as more monthly data on the quarter become available.

Popular examples of full system methods are common factor models and mixed frequency vector autoregressive (VAR) models. Common factor models are a way to exploit 
the whole available information set by condensing all time series into a few factors. One popular example is the construction of composite indicators (CI). These can be constructed for forecasting or for describing the current state of the economy. Stock and Watson (1990) construct a coincident index by applying maximum likelihood analysis to four monthly coincident indicators. Mariano and Murasawa (2003) extend this model by including quarterly GDP data and thus allowing for mixed frequency data. The model is cast into state space form and the likelihood is maximized using the Kalman Filter. In their model, all variables are driven by one unobserved monthly common factor and by an idiosyncratic factor for each individual series.

In the approach of Mariano and Murasawa (2003) the model operates at the highest frequency in the data, so all series are treated as monthly time series. All variables are assumed to be generated, but not necessarily observed, at this highest frequency, and thus can be used to produce forecasts of any variable at this frequency. The quarterly variable (GDP growth) is observed every third month and has missing values for the first two months of every quarter. This series is interpolated in order to estimate the unobserved latent monthly GDP. The suggested filtering algorithm is only an approximation. Aruoba, Diebold, and Scotti (2008) extend this model, but avoid approximations. They employ a one factor dynamic model to extract the unobserved state of the US economy, using four variables, including real high frequency (weekly and daily) data. The inclusion of high frequency daily data does not really change the picture compared with using only monthly indicators, but updates are available sooner.

Wohlrabe (2009) provides an extensive and detailed overview of the different models that have been explored using both of these approaches. Extensive descriptions of all model specifications and many empirical examples with comparisons are provided.

In this paper we propose a new method, in which the higher frequency data is stacked into a vector of observations which has the lower frequency. This is similar to the method proposed by Marcellino, Carriero, and Clark (2014). However, we propose a full system approach, in which the dynamics of all variables are described by the model. To discuss our methods in more specific terms, we consider a dynamic factor model, similar to the one proposed by Mariano and Murasawa (2003). We show that there is no need to introduce artificial missing values in the analysis while all the high frequency information is preserved and can still be analyzed in a computationally feasible way. Also the high frequency (monthly) optimal updating of new information can remain without the need to increase computational complexities.

Furthermore, we modify the method of maximum likelihood for parameter estimation by giving extra weight to one or more specific variables in a full system model approach. For example, when a dynamic factor model is adopted for the nowcasting and forecasting of a quarterly growth in gross domestic product (GDP), more weight can be given to GDP growth in comparison to the other variables in the dynamic factor model. For this purpose we introduce variable-specific weights in the likelihood function. We first discuss the asymptotic properties of this weighted maximum likelihood estimator and we 
show that the estimator is consistent and asymptotically normal. We further verify our new approach in a Monte Carlo study to investigate the effect of different choices for the weights in different scenarios. We also adopt the weighted likelihood function for parameter estimation in our empirical study concerning the nowcasting and forecasting of US GDP growth based on a full system dynamic factor model with mixed frequency variables.

The outline of the paper is as follows. In Section 2 we show how high frequency autoregressive models can be specified as observationally equivalent models with a lower frequency. This method is illustrated by means of univariate monthly autoregressive (AR) processes, which we then formulate as quarterly and yearly AR processes. In many cases these new formulations lead to computational gains. In Section 3 we show how these transformations can be used to simultaneously model variables with different frequencies. We describe the specifications of our new approach in detail and also describe the models of other approaches, which will be used as benchmark models in the empirical section. In Section 4 we present our weighted maximum likelihood approach that is used for the focusing on key variables within a full system model. Asymptotic properties of the resulting estimator are derived. We also explore its small-sample properties in a Monte Carlo study and discuss the effect of using different weights in different scenarios. In Section 5 we present and explore the results of our empirical study concerning US GDP growth. We compare the nowcasting and forecasting accuracies of our new approach with those of benchmark aggregation and interpolation methods. We also study the empirical relevance of weighted estimation. Section 6 summarizes and concludes.

\section{Low Frequency Solution for Univariate Models}

\subsection{Notation}

In this section we discuss a stacking approach for analyzing high frequency univariate observations in a low frequency vector model. We first consider the case of monthly data (high frequency) that is represented by quarterly (low frequency) vectors. We use the notation $x_{\tau}^{m}$ for a variable $x$ that is observed on a monthly $(m)$ basis with monthly time index $\tau$. The observations of the time series $x_{\tau}^{m}$ can be stacked into a quarterly $(q)$ observed $3 \times 1$ vector $x_{t}^{q}$ with quarterly time index $t$

$$
x_{t}^{q}=\left(\begin{array}{c}
x_{t, 1}^{q} \\
x_{t, 2}^{q} \\
x_{t, 3}^{q}
\end{array}\right)=\left(\begin{array}{c}
x_{3(t-1)+1}^{m} \\
x_{3(t-1)+2}^{m} \\
x_{3(t-1)+3}^{m}
\end{array}\right),
$$

where $x_{t, i}^{q}$ is the $i$-th element of $x_{t}^{q}$ with index $t$ indicating the number of the quarter of the observation and index $i$ indicating the number of the month within the quarter; we have $t=1, \ldots, n, i=1,2,3$ and $\tau=1, \ldots, 3 n$, since each quarter consists of $s=3$ months.

In a similar way, we can represent the monthly observations into yearly vectors. The 
monthly observed series $x_{\tau}^{m}$ can be stacked into a yearly $(y)$ observed $12 \times 1$ vector $x_{t}^{y}$ with yearly time index $t$. We then have

$$
x_{t}^{y}=\left(\begin{array}{c}
x_{t, 1}^{y} \\
x_{t, 2}^{y} \\
\vdots \\
x_{t, 12}^{y}
\end{array}\right)=\left(\begin{array}{c}
x_{12(t-1)+1}^{m} \\
x_{12(t-1)+2}^{m} \\
\vdots \\
x_{12(t-1)+12}^{m}
\end{array}\right) \text {, }
$$

where $x_{t, i}^{y}$ is the $i$-th element of $x_{t}^{y}$ with the first $t$ indicating the number of the year of the observation and second index $i$ indicating the number of the month within the year; we have $t=1, \ldots, n, i=1, \ldots, 12$ and $\tau=1, \ldots, 12 n$, since each year consists of $s=12$ months. Throughout this paper, we will use the superscripts $m, q$ and $y$ to indicate the frequency of time series; we only use this notation where we deem it is necessary. When a variable or vector has only one index, this index typically refers to the number of the month, quarter or year of the observation. The second index refers to the number of the month within the quarter or year of the observation.

\subsection{Linear State Space Models}

The general linear Gaussian state space model can be written in a variety of ways. In this paper we adopt the notation used in Durbin and Koopman (2012), where the model is given as

$$
\begin{aligned}
x_{t} & =Z \alpha_{t}+\epsilon_{t}, & & \epsilon_{t} \sim \operatorname{NID}(0, H), \\
\alpha_{t+1} & =T \alpha_{t}+R \eta_{t}, & & \eta_{t} \sim \operatorname{NID}(0, Q),
\end{aligned}
$$

where $x_{t}$ is a $k \times 1$ vector of observations called the observation vector and $\alpha_{t}$ is an unobserved $m \times 1$ vector called the state vector. The system matrices $Z, T, R, H$ and $Q$ are initially assumed to be known and the error terms $\epsilon_{t}$ and $\eta_{t}$ are assumed to be serially independent and independent of each other at all time points. In practice, some or all of the matrices $Z, T, R, H$ and $Q$ will depend on elements of an unknown parameter vector $\psi$.

In the state space model, the state vector $\alpha_{t}$ cannot be observed directly and hence we base the analysis on observations $x_{t}$. These equations hold true for any frequency, as long as the state vector has the same frequency as the observation vector. Therefore, we do not use a superscript to indicate the frequency of the series, although we use these mostly for low frequency models.

The initial state vector $\alpha_{1}$ is generated from $\mathrm{N}\left(a_{1}, P_{1}\right)$, independently of $\epsilon_{1}, \ldots, \epsilon_{n}$ and $\eta_{1}, \ldots, \eta_{n}$, where $a_{1}$ and $P_{1}$ are assumed known, although $P_{1}$ may depend on the parameter vector $\psi$. 


\subsection{Autoregressive Processes}

Autoregressive structures are the key ingredient for many dynamic linear models and therefore we will adopt autoregressive models to illustrate our stacking approach. We adopt our stacking method for other dynamic models in Section 3 as well.

Suppose that the univariate monthly observed variable $x_{\tau}^{m}$ is modeled by the autoregressive (AR) process with $p$ lagged dependent variables, that is

$$
x_{\tau}^{m}=\phi_{1} x_{\tau-1}^{m}+\phi_{2} x_{\tau-2}^{m}+\ldots+\phi_{p} x_{\tau-p}^{m}+\varepsilon_{\tau}^{m}, \quad \varepsilon_{\tau}^{m} \sim N I D\left(0, \sigma_{\varepsilon}^{2}\right),
$$

where $\phi_{1}, \ldots, \phi_{p}$ are fixed and unknown autoregressive coefficients for monthly lags and $\varepsilon_{\tau}^{m}$ represents a serially uncorrelated Gaussian disturbance sequence with zero mean and a fixed and unknown variance $\sigma_{\varepsilon}^{2}$.

In the remainder of this subsection we provide quarterly model specifications for monthly AR processes. Some derivations and the initial properties of the state vector are provided in Appendix A. The generalizations to yearly, or other low frequencies, model specifications are straightforward.

\section{AR(1) PROCESS}

The monthly observations from the $\mathrm{AR}(1)$ process $x_{\tau}^{m}=\phi x_{\tau-1}^{m}+\varepsilon_{\tau}^{m}$ are stacked into the quarterly $3 \times 1$ vector $x_{t}^{q}$ of (1). The quarterly process of the stacked variable $x_{t}^{q}$ is then given by the vector autoregressive process

$$
x_{t}^{q}=T x_{t-1}^{q}+R \varepsilon_{t}^{q}
$$

with

$$
T=\left(\begin{array}{ccc}
0 & 0 & \phi \\
0 & 0 & \phi^{2} \\
0 & 0 & \phi^{3}
\end{array}\right), \quad R=\left(\begin{array}{ccc}
1 & 0 & 0 \\
\phi & 1 & 0 \\
\phi^{2} & \phi & 1
\end{array}\right)
$$

such that the variance matrix of the vector $x_{t}^{q}$, conditional on $x_{t-1}^{q}$, is equal to $\sigma_{\varepsilon}^{2} R R^{\prime}$. We notice that all three elements of $x_{t}^{q}$ depend only on the last element of $x_{t-1}^{q}$ and on the associating elements of the vector of disturbances $\varepsilon_{t}^{q}$. The vector $\varepsilon_{t}^{q}$ is the result of stacking the values of $\varepsilon_{\tau}^{m}$ in similar fashion as in (1). The autoregressive process (5) is equal to the linear Gaussian state space model (3) with state vector $\alpha_{t}=x_{t}^{q}$ and with system matrices $T$ and $R$ given by (6) and $Z=I_{3}, H=0, Q=\sigma_{\varepsilon}^{2}$ and $\eta_{t}=\varepsilon_{t}^{q}$.

\section{AR(3) PROCESS}

The monthly observations from the $\mathrm{AR}(3)$ process $x_{\tau}^{m}=\phi_{1} x_{\tau-1}^{m}+\phi_{2} x_{\tau-2}^{m}+\phi_{3} x_{\tau-3}^{m}+\varepsilon_{\tau}^{m}$ are stacked into the quarterly $3 \times 1$ vector $x_{t}^{q}$ of (1). The quarterly process of the stacked variable $x_{t}^{q}$ is again given by the vector autoregressive process (5) with 


$$
\begin{aligned}
T & =\left(\begin{array}{ccc}
\phi_{3} & \phi_{2} & \phi_{1} \\
\phi_{1} \phi_{3} & \phi_{1} \phi_{2}+\phi_{3} & \phi_{1}^{2}+\phi_{2} \\
\phi_{1}^{2} \phi_{3}+\phi_{2} \phi_{3} & \phi_{1}^{2} \phi_{2}+\phi_{1} \phi_{3}+\phi_{2}^{2} & \phi_{1}^{3}+2 \phi_{1} \phi_{2}+\phi_{3}
\end{array}\right), \\
R & =\left(\begin{array}{ccc}
1 & 0 & 0 \\
\phi_{1} & 1 & 0 \\
\phi_{1}^{2}+\phi_{2} & \phi_{1} & 1
\end{array}\right)
\end{aligned}
$$

The variance matrix of the vector $x_{t}^{q}$, conditional on $x_{t-1}^{q}$, is equal to $\sigma_{\varepsilon}^{2} R R^{\prime}$. We notice that all three elements of $x_{t}^{q}$ depend on all three elements of $x_{t-1}^{q}$ and on the associating elements of the vector of disturbances $\varepsilon_{t}^{q}$. The state space formulation (3) has state vector $\alpha_{t}=x_{t}^{q}$ with $T$ and $R$ given by (7) and with system matrices $T$ and $R$ given by (6) and $Z=I_{3}, H=0, Q=\sigma_{\varepsilon}^{2}$ and $\eta_{t}=\varepsilon_{t}^{q}$.

For the $\operatorname{AR}(2)$ process, matrix $T$ is the same as in (7) but with $\phi_{3}=0$, such that its first column reduces to a zero vector and $R$ is the same matrix as for the $\operatorname{AR}(3)$ process in (7).

\section{AR(4) PROCESS}

For $\operatorname{AR}(p)$ processes of order $p>3$ in the state space formulation, the state vector in (3) is extended with more lags of $x_{t}^{q}$. In case of the $\operatorname{AR}(4)$ process, we have $\alpha_{t}=$ $\left(x_{t-1,3}^{q}, x_{t, 1}^{q}, x_{t, 3}^{q}, x_{t, 3}^{q}\right)^{\prime}$ and we have

$$
T=\left(\begin{array}{cccc}
0 & 0 & 0 & 1 \\
\phi_{4} & \phi_{3} & \phi_{2} & \phi_{1} \\
\phi_{1} \phi_{4} & \phi_{1} \phi_{3}+\phi_{4} & \phi_{1} \phi_{2}+\phi_{3} & \phi_{1}^{2}+\phi_{2} \\
\phi_{1}^{2} \phi_{4}+\phi_{2} \phi_{4} & \phi_{1}^{2} \phi_{3}+\phi_{2} \phi_{3}+\phi_{1} \phi_{4} & \phi_{1}^{2} \phi_{2}+\phi_{1} \phi_{3}+\phi_{2}^{2}+\phi_{4} & \phi_{1}^{3}+2 \phi_{1} \phi_{2}+\phi_{3}
\end{array}\right),
$$

where the variance matrix of the process is defined as $\sigma_{\varepsilon}^{2} R R^{\prime}$ with

$$
R=\left(\begin{array}{cccc}
0 & 0 & 0 & 0 \\
0 & 1 & 0 & 0 \\
0 & \phi_{1} & 1 & 0 \\
0 & \phi_{1}^{2}+\phi_{2} & \phi_{1} & 1
\end{array}\right)
$$

For higher order $\operatorname{AR}(p)$ processes the same principle can be used. The state vector $\alpha_{t}$ has to be extended with the necessary lags of $x_{t}^{q}$ and rows and columns with ones and zeros need to be added to the matrices $T$ and $R$.

\subsection{Computing Times}

The $\operatorname{AR}(p)$ models can be specified as a linear Gaussian state space model (3) as we have illustrated in the previous section. Whether the monthly AR model is represented as the 
monthly process $x_{\tau}^{m}$, or as the stacked quarterly $3 \times 1$ vector $x_{t}^{q}$ or as the yearly $12 \times 1$ vector $x_{t}^{y}$ has no effect on the value of the loglikelihood function for given parameters $\phi_{1}, \ldots, \phi_{p}$ and $\sigma_{\varepsilon}^{2}$. The different model representations are observationally equivalent. In all cases, the Kalman filter can be used for likelihood evaluation. Hence the maximized loglikelihood value and the corresponding parameter estimates are the same for each case.

However, the representation has an effect on computing times. For example, when the data are treated as monthly observations, we have $n=12,000$. When the data is stacked into quarterly $3 \times 1$ vectors we have $n=4,000$ and with yearly $12 \times 1$ vectors we only have $n=1,000$. Furthermore, with different frequencies we have different state vector lengths, and hence the transition matrix $T$ and the variance matrix $\sigma_{\varepsilon}^{2} R R^{\prime}$ of the state vector have different dimensions for the different frequencies. The different dimensions will have a clear effect on the computations for the loglikelihood evaluation.

To illustrate this, we have evaluated the loglikelihood value 10,000 times for $\operatorname{AR}(p)$ models of different orders $p$ and using the three different representations: monthly, quarterly and yearly. For each $\operatorname{AR}(p)$ process the calculations were performed on a time series with an $\operatorname{AR}(p)$ data generating process, consisting of 12,000 monthly observations. The loglikelihood value was calculated 10,000 times using the parameter values that maximized the loglikelihood function. We have verified that all likelihood evaluations resulted in the same value.

The computing times for the different combinations of $\operatorname{AR}(p)$ processes and frequencies are shown in Table 1. It is clear that for $\mathrm{AR}(1)$ and $\mathrm{AR}(2)$ processes, the structure with monthly single observations is most efficient. In these situations the smaller state vector outweighs the fact that the Kalman Filter has to go through 12,000 iterations instead of 4,000 and 1,000 for the quarterly and yearly structure, respectively. For $\operatorname{AR}(3)$ and higher orders, the quarterly structure is faster than the monthly. This can be explained by the fact that both structures have the same vector length for $\operatorname{AR}(3)$ and higher but the time dimension is three times smaller in the quarterly structure. For $\mathrm{AR}(10)$ and higher orders, the yearly structure is the fastest. For these orders the smaller time dimension starts to outweigh the larger state vector and for $\mathrm{AR}(12)$ and higher, all three structures have the same vector length, due to the number of lags that has to be included in the state vector in the monthly and quarterly structure. This example clearly illustrates that stacking observations into vectors with a lower frequency can lead to large gains in computational efficiency, especially when many lags of the observations are included in the model. 
Table 1: Computing times

\begin{tabular}{cccccccc}
\hline & \multicolumn{2}{c}{ Computing time (in seconds) } & & \multicolumn{3}{c}{ State vector length } \\
\cline { 2 - 3 } \cline { 6 - 8 } & $\begin{array}{c}\text { Monthly } \\
(n=12 \mathrm{k})\end{array}$ & $\begin{array}{c}\text { Quarterly } \\
(n=4 \mathrm{k})\end{array}$ & $\begin{array}{c}\text { Yearly } \\
(n=1 \mathrm{k})\end{array}$ & & $\begin{array}{c}\text { Monthly } \\
(n=12 \mathrm{k})\end{array}$ & $\begin{array}{c}\text { Quarterly } \\
(n=4 \mathrm{k})\end{array}$ & $\begin{array}{c}\text { Yearly } \\
(n=1 \mathrm{k})\end{array}$ \\
\hline 1 & 10 & 13 & 61 & & 1 & 3 & 12 \\
2 & 11 & 16 & 67 & & 2 & 3 & 12 \\
3 & 26 & 18 & 76 & & 3 & 3 & 12 \\
4 & 41 & 27 & 85 & & 4 & 4 & 12 \\
5 & 59 & 40 & 92 & & 5 & 5 & 12 \\
6 & 83 & 56 & 100 & & 6 & 6 & 12 \\
7 & 106 & 73 & 108 & & 7 & 7 & 12 \\
8 & 129 & 90 & 116 & & 8 & 8 & 12 \\
9 & 154 & 111 & 124 & & 9 & 9 & 12 \\
10 & 191 & 137 & 133 & & 10 & 10 & 12 \\
11 & 226 & 162 & 139 & & 11 & 11 & 12 \\
12 & 265 & 190 & 146 & & 12 & 12 & 12 \\
\hline
\end{tabular}

The left panel of this table presents the average computing time (in seconds) that is required to evaluate the loglikelihood function for an $\operatorname{AR}(p)$ model of order $p$ for a monthly time series $x_{\tau}^{m}$ that is generated by an $\operatorname{AR}(p)$ model. Three different approaches are used: treating the data as monthly observations, stacking the data into quarterly $3 \times 1$ vectors and stacking the data into yearly $12 \times 1$ vectors. Each value represents the average over 10,000 simulation runs. For each value of $p$ the fastest of the three approaches is highlighted. The right panel of this table presents the state vector length for each scenario.

\section{Mixed Frequency Dynamic Factor Models}

In the previous section we have shown that in some situations computing times can be reduced by stacking the monthly data into observation vectors with a lower frequency. This is especially the case when many lags of the dependent variable are included in the model. Another situation in which stacking the data into vectors with a lower frequency is very convenient is when we simultaneously analyze variables which are observed at different frequencies. We then may stack the series observed at higher frequencies into vectors of the lowest frequency at which one of the variables is observed.

Suppose we have a monthly observed series $x_{\tau}^{m}$, which is modeled by the $\operatorname{AR}(1)$ process (4) with $p=1$, that is $x_{\tau}^{m}=\phi_{x} x_{\tau-1}^{m}+\varepsilon_{\tau}^{m}$ with the autoregressive parameter $\phi_{x}$. We also have a quarterly observed series $y_{t}$, which is modeled by the AR(1) process as given by $y_{t}=\phi_{y} y_{t-1}+\xi_{t}$, with $\xi_{t} \sim N I D\left(0, \sigma_{\xi}^{2}\right)$, where $\phi_{y}$ is the autoregressive coefficient for the quarterly lagged dependent variable $y_{t-1}$ and $\xi_{t}$ is the Gaussian disturbance that is possibly correlated with $\varepsilon_{t}^{q}$ in the formulation of (5) for $x_{\tau}^{m}$. We do not use the superscript $q$ for $y_{t}$ and $\xi_{t}$, because we assume throughout this section that they are specifically quarterly variables for which no monthly values become available.

When we stack the values of $x_{\tau}^{m}$ into quarterly $3 \times 1$ vectors $x_{t}^{q}$, as we have discussed in Section 2, then the two processes for $y_{t}$ and $x_{t}^{q}$ can be combined into a low frequency 
multivariate process

$$
\left(\begin{array}{c}
y_{t+1} \\
x_{t+1,1}^{q} \\
x_{t+1,2}^{q} \\
x_{t+1,3}^{q}
\end{array}\right)=\left(\begin{array}{cccc}
\phi_{y} & 0 & 0 & 0 \\
0 & 0 & 0 & \phi_{x} \\
0 & 0 & 0 & \phi_{x}^{2} \\
0 & 0 & 0 & \phi_{x}^{3}
\end{array}\right)\left(\begin{array}{c}
y_{t} \\
x_{t, 1}^{q} \\
x_{t, 2}^{q} \\
x_{t, 3}^{q}
\end{array}\right)+\left(\begin{array}{cccc}
1 & 0 & 0 & 0 \\
0 & 1 & 0 & 0 \\
0 & \phi_{x} & 1 & 0 \\
0 & \phi_{x}^{2} & \phi_{x} & 1
\end{array}\right)\left(\begin{array}{c}
\xi_{t} \\
\varepsilon_{t, 1}^{q} \\
\varepsilon_{t, 2}^{q} \\
\varepsilon_{t, 3}^{q}
\end{array}\right)
$$

for $t=1, \ldots, n$. We notice the difference between the autoregressive parameters $\phi_{x}$ and $\phi_{y}$. The parameter $\phi_{x}$ measures the dependence of $x_{\tau}$ on its lagged value $x_{\tau-1}$ of one month earlier, whereas the parameter $\phi_{y}$ indicates the dependence of $y_{t}$ on its lagged value $y_{t-1}$ of one quarter earlier.

In the example above the two processes $y_{t}$ and $x_{t}^{q}$ are independent of each other, but the zeros in the first row and/or column of the matrix $R$ can be replaced by non-zero values to include covariances between $\xi_{t}$ and $\varepsilon_{t}^{q}$. These can be included if there is a relation between the contemporaneous values of $y_{t}$ and $x_{t}^{q}$. Furthermore, the first row and column of the transition matrix $T$ can be used to include lagged dependencies between the two series. The first row can be used when $y_{t}$ is dependent on $x_{t-1}^{q}$ and the first column if the values of $x_{t}^{q}$ depend on the value of $y_{t-1}$. If other dynamic dependencies need to be introduced, we can extend formulations such as (10) straightforwardly.

\subsection{Dynamic FaCtor Models}

It is an increasingly common practice to simultaneously model $y_{t}$ and $x_{\tau}^{m}$ by means of an unobserved common dynamic factor. The typical example is to have a large vector of $x_{\tau}^{m}$ variables that are potentially useful for the forecasting of $y_{t}$. For illustrative purposes, we initially assume that $x_{\tau}^{m}$ is a single variable. We then introduce a dynamic factor with a monthly frequency and we denote this factor by $f_{\tau}^{m}$. The monthly variable $x_{\tau}^{m}$ has loading $\beta_{x}$ on this common factor. This process can be described as

$$
x_{\tau}^{m}=\beta_{x} f_{\tau}^{m}+\varepsilon_{\tau}^{m}, \quad \varepsilon_{\tau}^{m} \sim N I D\left(0, \sigma_{\varepsilon}^{2}\right),
$$

where the common factor $f_{\tau}^{m}$ is specified by an $\operatorname{AR}(p)$ process, using the formulation

$$
f_{\tau}^{m}=\phi_{f, 1} f_{\tau-1}^{m}+\phi_{f, 2} f_{\tau-2}^{m}+\ldots+\phi_{f, p} f_{\tau-p}^{m}+\eta_{\tau}^{m}, \quad \eta_{\tau}^{m} \sim N I D\left(0, \sigma_{\eta}^{2}\right)
$$

The quarterly variable $y_{t}$ is also loaded on the common factor $f_{\tau}^{m}$, with loading $\beta_{y}$ on all three values of $f_{\tau}^{m}$ within the quarter $t$. This can be described as

$$
\begin{aligned}
y_{t} & =\beta_{y} f_{(3 t-2)}^{m}+\beta_{y} f_{(3 t-1)}^{m}+\beta_{y} f_{(3 t)}^{m}+\xi_{t} & \\
& =\left(\begin{array}{lll}
\beta_{y} & \beta_{y} & \beta_{y}
\end{array}\right) f_{t}^{q}+\xi_{t}, & \xi_{t} \sim N I D\left(0, \sigma_{\xi}^{2}\right),
\end{aligned}
$$

where $f_{t}^{q}$ is the $3 \times 1$ vector of stacked observations of the process $f_{\tau}^{m}$ using the stacking approach that was described for the series $x_{\tau}^{m}$ in (1).

Hence, the simultaneous process for $y_{t}$ and $x_{\tau}^{m}$ can be described by a process in which 
the values of $x_{\tau}^{m}$ and $f_{\tau}^{m}$ are stacked into the quarterly $3 \times 1$ vectors $x_{t}^{q}$ and $f_{t}^{q}$

$$
\left(\begin{array}{c}
y_{t} \\
x_{t, 1}^{q} \\
x_{t, 2}^{q} \\
x_{t, 3}^{q}
\end{array}\right)=\left(\begin{array}{ccc}
\beta_{y} & \beta_{y} & \beta_{y} \\
\beta_{x} & 0 & 0 \\
0 & \beta_{x} & 0 \\
0 & 0 & \beta_{x}
\end{array}\right)\left(\begin{array}{c}
f_{t, 1}^{q} \\
f_{t, 2}^{q} \\
f_{t, 3}^{q}
\end{array}\right)+\left(\begin{array}{c}
\xi_{t} \\
\varepsilon_{t, 1}^{m} \\
\varepsilon_{t, 2}^{m} \\
\varepsilon_{t, 3}^{m}
\end{array}\right)
$$

where the variance matrix of the disturbances is a $4 \times 4$ diagonal matrix with the first diagonal element equal to $\sigma_{\xi}^{2}$ and the other three diagonal elements equal to $\sigma_{\varepsilon}^{2}$. The vector autoregressive process for $f_{t}^{q}$ is given by

$$
f_{t+1}^{q}=T_{f} f_{t}^{q}+R_{f} \eta_{t}^{q}, \quad \eta_{t}^{q} \sim N I D\left(0, \sigma_{\eta}^{2}\right),
$$

It is straightforward to generalize the model for $y_{t}$ by having different loadings $\beta_{y, 1}, \beta_{y, 2}$ and $\beta_{y, 3}$ for the three values of $f_{t}^{q}$ within the quarter associated with $t$. Additional (monthly and quarterly) variables can also be included into the model and the model can be extended with specific factors for specific variables. The generality of the state space framework of Section 2.2 can be fully exploited.

We further can generalize the current specification by considering $x_{\tau}^{m}$ as a $k \times 1$ vector of variables. Mariano and Murasawa (2003) analyze such a dynamic factor model with one quarterly observed variable (in their case GDP growth) and four $(k=4)$ monthly observed economic indicators. In their analysis, they extend the Stock and Watson (1990) coincident index for business cycles, which uses four monthly indicators, with quarterly observed real GDP growth. Here we adopt this modeling framework but our analysis is based on the low frequency stacking approach as indicated above. In the empirical illustration of Section 5 we investigate different approaches for the simultaneous modeling of the five time series with a mix of quarterly and monthly frequencies.

First, in Section 3.2 we discuss the original solution by Mariano and Murasawa (2003) in treating mixed frequency data; they analyze all series at the highest frequency. In this approach, artificial missing values are introduced for the series that is observed at the lower frequency and interpolation techniques are used to describe the dynamics of the unobserved 'latent' monthly GDP growth. In Section 3.3 we explore a second approach where all series are modeled at the lowest frequency by aggregating the high frequency series to quarterly totals. In this approach, no artificial missing values are needed, but information about the high frequency series is lost and the model does not allow the econometrician to address high frequency dynamics. Finally, in Section 3.4 we discuss our model in which the high frequency series are stacked into vectors of observations at the lower frequency, as discussed in Section 2. In this approach, no artificial missing values are needed and also no information is lost about the high frequency series and their dynamic features. 


\subsection{IntERPolation ApProAch}

In the mixed frequency interpolation (MFI) approach, all series are treated at the highest frequency, say as monthly time series. All variables are driven by one unobserved monthly common factor $f_{\tau}$ and by the idiosyncratic factors $u_{\tau}$ and $v_{\tau}$. The variable quarterly $y_{t}$ is observed every third month and has missing values for the first two months of every quarter. Therefore, the data matrix has the following structure

$$
\left[\begin{array}{ccccccccc}
\cdot & \cdot & y_{3} & \cdot & \cdot & y_{6} & \cdot & \ldots & y_{3 n} \\
x_{1} & x_{2} & x_{3} & x_{4} & x_{5} & x_{6} & x_{7} & \ldots & x_{3 n}
\end{array}\right]
$$

where $x_{\tau}$ is the $k \times 1$ vector $\left(x_{\tau}^{(1)}, \ldots, x_{\tau}^{(k)}\right)^{\prime}$ and $n$ is the number of quarters in the sample period. Since in this approach all series are treated as monthly series, we choose to drop the superscript $m$, in order to avoid cumbersome notation. The contemporaneous and dynamic interactions between $y_{\tau}$ and the vector of monthly observed variables $x_{\tau}$ are specified via the model

$$
\left(\begin{array}{c}
\tilde{y}_{\tau}^{m} \\
x_{\tau}
\end{array}\right)=\left(\begin{array}{c}
\beta_{y} g\left(f_{\tau}\right) \\
\beta_{x} f_{\tau}
\end{array}\right)+\left(\begin{array}{c}
g\left(u_{\tau}\right) \\
v_{\tau}
\end{array}\right)+\left(\begin{array}{c}
\xi_{\tau} \\
\varepsilon_{\tau}
\end{array}\right)
$$

where $\tilde{y}_{\tau}^{m}$ is the latent monthly variable for $y$ and for which we only have observations available in the last month of each quarter, $\beta_{y}$ is a scalar coefficient, $\beta_{x}$ is a $k \times 1$ vector of coefficients, and

$$
g\left(a_{\tau}\right)=\frac{1}{3} a_{\tau}+\frac{2}{3} a_{\tau-1}+a_{\tau-2}+\frac{2}{3} a_{\tau-3}+\frac{1}{3} a_{\tau-4}
$$

for $\tau=1, \ldots, 3 n$. The vector $u_{\tau}$ is the stationary sequence of the idiosyncratic factor for $y_{\tau}$ and $v_{\tau}$ is the stationary sequence of the vector of idiosyncratic factors for $x_{\tau}$ and consists of one value per month for each monthly observed variable $x_{\tau}^{(i)}$.

The factors $f_{\tau}, u_{\tau}$ and $v_{\tau}$ are modeled as AR processes

$$
f_{\tau} \sim \operatorname{AR}\left(p_{f}\right), \quad u_{\tau} \sim \operatorname{AR}\left(p_{u}\right), \quad v_{\tau} \sim \operatorname{AR}\left(p_{v}\right),
$$

where $\operatorname{AR}(p)$ refers to the process in (4) with order $p$ that can be different, that is $p=p_{f}, p_{u}, p_{v}$ respectively. There are no interactions between the series of $f_{\tau}, u_{\tau}$ and $v_{\tau}$ nor between the series of $v_{\tau}^{(i)}$ and $v_{\tau}^{(j)}$ for any $i \neq j$.

We have established the model by Mariano and Murasawa (2003) and implicitly their model-based solution to the mixed frequency problem. They advocate to use the Kalman filter for likelihood evaluation and general analysis. Specifically, they take advantage of the fact that the Kalman filter can treat missing observations without a problem.

\subsection{Aggregation Approach}

An alternative approach, where the introduction of artificial missing values is not required, is the aggregation of the monthly series $x_{\tau}^{m}$ into quarterly totals $\bar{x}_{t}^{q}$ and the treatment of 
all series as quarterly series. This model then only describes quarterly dynamics. The unobserved common factor must also become quarterly. To avoid cumbersome notation we drop the superscript $q$ and we write the model in the form

$$
\left(\begin{array}{c}
y_{t} \\
\bar{x}_{t}
\end{array}\right)=\left[\begin{array}{ccc}
\beta_{y} & 1 & 0 \\
\beta_{x} & 0 & I_{k}
\end{array}\right]\left(\begin{array}{c}
f_{t} \\
u_{t} \\
\bar{v}_{t}
\end{array}\right)+\left(\begin{array}{c}
\xi_{t} \\
\bar{\varepsilon}_{t}
\end{array}\right),
$$

for $t=1, \ldots, n$, where $\bar{x}_{t}$ and $\beta_{x}$ are both $k \times 1$ vectors. The common factor $f_{t}$ and the idiosyncratic factors $u_{t}$ and $\bar{v}_{t}$ can still be modeled by AR processes as in (19). However, we must take care when interpreting the values of the parameters of these processes, as they now describe the dynamics from quarter to quarter. We will hereafter refer to this approach as the mixed frequency aggregation (MFA) approach.

\subsection{Stacking Approach}

Here we introduce the stacking method of Section 2 for the mixed frequency dynamic factor model in the spirit of Mariano and Murasawa (2003). We consider for each quarter the observed values of $y_{t}$ and $x_{t}^{q}$, where $x_{t}^{q}$ consists of three stacked observations of the monthly vector variable $x_{\tau}^{m}$, which we define as the $k \times 1$ vector $\left(x_{\tau}^{(1)}, \ldots, x_{\tau}^{(k)}\right)^{\prime}$. In this approach, no artificial missing values are needed and no information is lost regarding the high frequency series and high frequency dynamics. When we drop the superscript $q$ from the stacked vector $x_{t}^{q}$, we obtain the following model

$$
\left(\begin{array}{c}
y_{t} \\
x_{t, 1}^{(1)} \\
x_{t, 2}^{(1)} \\
x_{t, 3}^{(1)} \\
\vdots \\
x_{t, 1}^{(k)} \\
x_{t, 2}^{(k)} \\
x_{t, 3}^{(k)}
\end{array}\right)=\left[\begin{array}{ccccccccccc}
\beta_{y} & \beta_{y} & \beta_{y} & 1 & 0 & 0 & 0 & \ldots & 0 & 0 & 0 \\
\beta_{x^{(1)}} & 0 & 0 & 0 & 1 & 0 & 0 & \ldots & 0 & 0 & 0 \\
0 & \beta_{x^{(1)}} & 0 & 0 & 0 & 1 & 0 & \ldots & 0 & 0 & 0 \\
0 & 0 & \beta_{x^{(1)}} & 0 & 0 & 0 & 1 & \ldots & 0 & 0 & 0 \\
& \vdots & & & \vdots & & \ddots & & \vdots \\
\beta_{x^{(k)}} & 0 & 0 & 0 & 0 & 0 & 0 & \ldots & 1 & 0 & 0 \\
0 & \beta_{x^{(k)}} & 0 & 0 & 0 & 0 & 0 & \ldots & 0 & 1 & 0 \\
0 & 0 & \beta_{x^{(k)}} & 0 & 0 & 0 & 0 & \ldots & 0 & 0 & 1
\end{array}\right]\left(\begin{array}{c}
f_{t, 1}^{q} \\
f_{t, 2}^{q} \\
f_{t, 3}^{q} \\
u_{t} \\
v_{t, 1}^{(1)} \\
v_{t, 2}^{(1)} \\
v_{t, 3}^{(1)} \\
\vdots \\
v_{t, 1}^{(k)} \\
v_{t, 2}^{(k)} \\
v_{t, 3}^{(k)}
\end{array}\right)+\left(\begin{array}{c}
\xi_{t} \\
\varepsilon_{t, 1}^{(1)} \\
\varepsilon_{t, 2}^{(1)} \\
\varepsilon_{t, 3}^{(1)} \\
\vdots \\
\varepsilon_{t, 1}^{(k)} \\
\varepsilon_{t, 2}^{(k)} \\
\varepsilon_{t, 3}^{(k)}
\end{array}\right)
$$

for $t=1, \ldots, n$. Each vector series $x_{t}^{(i)}$ and $v_{t}^{(i)}$ have dimension $3 \times 1$ vectors; more formally we can write $x_{t}^{(i)}=\left(x_{t, 1}^{(i)}, x_{t, 2}^{(i)}, x_{t, 3}^{(i)}\right)^{\prime}$ and $v_{t}^{(i)}=\left(v_{t, 1}^{(i)}, v_{t, 2}^{(i)}, v_{t, 3}^{(i)}\right)^{\prime}$. The quarterly observed $y_{t}$ has the same loadings $\beta_{y}$ on all three elements of $f_{t}^{q}$, which is the $3 \times 1$ vector of stacked values of the monthly unobserved dynamic factor $f_{\tau}^{m}$. Each element of $x_{t}^{(i)}$ depends on the corresponding element of the vector $f_{t}^{q}$ with loading $\beta_{x^{(i)}}$, for $i=1, \ldots, k$.

We can also opt for a quarterly unobserved common factor $f_{t}$ so that we only have one 
value for each quarter, for all three months. In this case, we do not use the superscript $q$ and the model equation becomes

$$
\left(\begin{array}{c}
y_{t} \\
x_{t, 1}^{(1)} \\
x_{t, 2}^{(1)} \\
x_{t, 3}^{(1)} \\
\vdots \\
x_{t, 1}^{(k)} \\
x_{t, 2}^{(k)} \\
x_{t, 3}^{(k)}
\end{array}\right)=\left[\begin{array}{ccccccccc}
\beta_{y} & 1 & 0 & 0 & 0 & \ldots & 0 & 0 & 0 \\
\beta_{x^{(1)}} & 0 & 1 & 0 & 0 & \ldots & 0 & 0 & 0 \\
\beta_{x^{(1)}} & 0 & 0 & 1 & 0 & \ldots & 0 & 0 & 0 \\
\beta_{x^{(1)}} & 0 & 0 & 0 & 1 & \ldots & 0 & 0 & 0 \\
\vdots & & \vdots & & \ddots & & \vdots & \\
\beta_{x^{(k)}} & 0 & 0 & 0 & 0 & \ldots & 1 & 0 & 0 \\
\beta_{x^{(k)}} & 0 & 0 & 0 & 0 & \ldots & 0 & 1 & 0 \\
\beta_{x^{(k)}} & 0 & 0 & 0 & 0 & \ldots & 0 & 0 & 1
\end{array}\right]\left(\begin{array}{c}
f_{t} \\
u_{t} \\
v_{t, 1}^{(1)} \\
v_{t, 2}^{(1)} \\
v_{t, 3}^{(1)} \\
v_{t, 1}^{(2)} \\
\vdots \\
v_{t, 3}^{(k)}
\end{array}\right)+\left(\begin{array}{c}
\xi_{t} \\
\varepsilon_{t, 1}^{(1)} \\
\varepsilon_{t, 2}^{(1)} \\
\varepsilon_{t, 3}^{(1)} \\
\varepsilon_{t, 1}^{(2)} \\
\vdots \\
\varepsilon_{t, 3}^{(k)}
\end{array}\right)
$$

where $f_{t}$ is now a univariate quarterly dynamic factor. Here the quarterly observed variable $y_{t}$ depends on the factor with loading $\beta_{y}$ while each element of $x_{t}^{(i)}$ also depends on $f_{t}$ and with the same loading $\beta_{x^{(i)}}$, for $i=1, \ldots, k$. We will hereafter refer to our solution as the Mixed Frequency Stacking (MFS) method. The former case of (21) has a monthly dynamic factor and is labelled as MFS-M while the latter case of (22) has a quarterly factor and is labelled as MFS-Q.

For both MFS-M and MFS-Q models, the dynamic factor $f_{t}$ and the idiosyncratic factors $u_{t}$ and $v_{t}$ are modeled as AR processes as in (19). There are no interactions between the series of $f_{t}, u_{t}$ and $v_{t}$ nor between the series of $v_{t}^{(i)}$ and $v_{t}^{(j)}$ for any $i \neq j$.

\subsection{Generalizations}

The mixed frequency dynamic factor model with monthly observed variables $x_{\tau}^{m}$ that is stacked into the quarterly variable $x_{t}^{q}$ and a quarterly observed variable $y_{t}$, is a specific example in which our stacking approach can be used to simultaneously model variables with mixed frequencies. We emphasize that the use of the stacking approach is not limited to this particular example. The approach can handle multiple variables with multiple different frequencies, as long as the observations of all variables can be stacked into vectors that have the lowest frequency. For example, if one or more yearly observed variables $z_{t}^{y}$ would need to be added to the model, then all variables would have to be stacked into yearly vectors of observations. It is also possible to include variables with higher frequencies, such as daily or weekly. It only requires a somewhat more elaborate representation in state space form.

\section{Weighted Maximum Likelihood Estimator}

Next we introduce a weighted likelihood function for the mixed frequency dynamic factor model and we develop the asymptotic properties of the estimator that maximizes the weighted likelihood function. In this section we consider the basic dynamic factor model (11), (12) and (13) where both $y_{t}$ and $x_{\tau}^{m}$ can be treated as vectors. The same 
developments can be easily adapted for more general dynamic factor specifications. The loglikelihood function for the model can be given by

$$
\mathcal{L}_{T}\left(\psi, f_{1}^{m}\right):=\log p(y, x ; \psi)=\log p(y \mid x ; \psi)+\log p(x ; \psi),
$$

where $p$ is the Gaussian density function, stacked vectors $y$ and $x$ collect all $T$ observations available for the variables $y_{t}$ and $x_{\tau}^{m}$ in the sample, respectively, the initial value of the monthly unobserved dynamic factor $f_{1}^{m}$ is treated as a fixed value, parameter vector $\psi$ collects all unknown coefficients in the model including the factor loadings $\beta_{x}$ and $\beta_{y}$, autoregressive parameters for the AR process, and the variances of the disturbances. The loglikelihood expression shows that the joint density can alternatively be expressed by a conditional density multiplied by a marginal density. For our purpose this expression is useful as it highlights the different roles of $y$ and $x$ : the variable $y_{t}$ is our key variable for which we require accurate model-based forecasts while the variables represented by $x_{\tau}^{m}$ are typically instrumental to improve the nowcasts and forecasts of $y_{t}$.

Under the assumption that $y$ and $x$ are jointly generated by a Gaussian dynamic factor model such as (11), (12) and (13), we can apply the Kalman filter to evaluate the loglikelihood function via the prediction error decomposition. Koopman and Durbin (2000) discuss an alternative filtering method in which each element of the observation vector $\left(y^{\prime}, x^{\prime}\right)^{\prime}$ is brought one at a time into the updating step of the Kalman filter. In effect, the vector series is converted into a univariate time series where multiple observations are available for the same time index. In our application, it means that for each available observation, whether it is for a monthly or a quarterly variable, an update takes place. It results in a truly online and high frequency filtering method even though the model is formulated in terms of a low frequency time index.

The Kalman filter is applied to the low frequency model with the (quarterly) time index $t$ and for the $r_{t} \times 1$ observation vector $z_{t}:=\left(y_{t}, x_{t}^{\prime}\right)^{\prime}$ where $x_{t}$ is the stacked vector of monthly variables $x_{\tau}^{m}$ within the quarter associated with time $t$, for $t=1, \ldots, n$. Hence the total number of observations is $T=\sum_{t=1}^{n} r_{t}$. The loglikelihood function is then given by

$$
\mathcal{L}_{T}\left(\psi, f_{1}^{m}\right)=-\frac{T}{2} \log (2 \pi)-\frac{1}{2} \sum_{t=1}^{n} \ell_{t}\left(z_{t} \mid \tilde{f}_{t}\left(\tilde{f}_{1}\right) ; \psi\right),
$$

where $\ell_{t}$ is the predictive Gaussian logdensity contribution at time $t$ and $\tilde{f}_{t}\left(\tilde{f}_{1}\right)$ is the predictor of the unobserved factor $f_{t}^{m}$ conditional on past observations $z_{1}, \ldots, z_{t-1}$ and its initial value $\tilde{f}_{1}=f_{1}^{m}$. The Kalman filter evaluates recursively $\tilde{f}_{t}\left(\tilde{f}_{1}\right)$ from which $\ell_{t}$ can be evaluated. In spirit of the likelihood decomposition in (23), likelihood evaluation via the method of Koopman and Durbin (2000) is partly based on

$$
\mathcal{L}_{T}\left(\psi, f_{1}^{m}\right)=-\frac{T}{2} \log (2 \pi)-\frac{1}{2} \sum_{t=1}^{n} \ell_{t}\left(y_{t} \mid \tilde{f}_{t}\left(\tilde{f}_{1}\right) ; \psi\right)+-\frac{1}{2} \sum_{t=1}^{n} \ell_{t}\left(x_{t} \mid y_{t}, \tilde{f}_{t}\left(\tilde{f}_{1}\right) ; \psi\right),
$$

which facilitates a separate treatment of $y_{t}$ and $x_{t}$. 
Maximum likelihood estimation for the parameter vector $\psi$ is based on applying a numerical quasi-Newton optimization method for the maximization of $\mathcal{L}_{T}\left(\psi, f_{1}^{m}\right)$, with respect to $\psi$. The maximization is an iterative process. After convergence, the maximum likelihood estimate of $\psi$ is obtained. For each iteration in this process, various loglikelihood evaluations are required and they are carried out by the Kalman filter. In the context of the mixed frequency dynamic factor model, the treatment of the observations in $z_{t}$ for the construction of the likelihood function is implied by the dynamic factor model. However, it is very likely that the dynamic factor model is misspecified as a model representation of the true data generation process for the variables represented in $z_{t}$. When our primary aim is to analyze $y_{t}$ in particular, we may be less concerned with the misspecification of $x_{t}$, to some extent. To reflect the higher importance of $y_{t}$ in comparison to $x_{t}$ in the likelihood construction for the misspecified dynamic factor model, we propose to give different weights to the likelihood contributions of $y_{t}$ and $x_{t}$ explicitly. Hence we propose the weighted loglikelihood function

$$
\mathcal{L}_{T}\left(\psi, w, f_{1}^{m}\right)=-\frac{T}{2} \log (2 \pi)-\frac{W}{2} \sum_{t=1}^{n} \ell_{t}\left(y_{t} \mid \tilde{f}_{t}\left(\tilde{f}_{1}\right) ; \psi\right)+-\frac{1}{2} \sum_{t=1}^{n} \ell_{t}\left(x_{t} \mid y_{t}, \tilde{f}_{t}\left(\tilde{f}_{1}\right) ; \psi\right),
$$

for a fixed and predetermined weight $W \geq 1$ and with $w:=W^{-1} \in[0,1]$. The weight $W$ is conveniently used in our Monte Carlo and empirical studies below while it is more appropriate to work with the inverse weight $w$ in the asymptotic theory that is developed next. The construction of the weighted loglikelihood function does not need further modifications. The estimator of $\psi$ that maximizes (26) is referred to as the weighted maximum likelihood (WML) estimator.

\subsection{Asymptotic Properties of the WML Estimator}

The properties of the weighted maximum likelihood estimator are derived for any choice of weight $w:=W^{-1} \in[0,1]$. We show that, when the model is correctly specified, then the WML estimator $\hat{\psi}_{T}(w)$ is consistent and asymptotically normal for the true parameter vector $\psi_{0} \in \Psi$. When the model is misspecified, we show that $\hat{\psi}_{T}(w)$ is consistent and asymptotically normal for a pseudo-true parameter $\psi_{0}^{*}(w) \in \Psi$ that minimizes a transformed Kullback-Leibler (KL) divergence between the true probability measure of the data and the measure implied by the model. We show that the transformed KL divergence takes the form of a pseudo-metric that gives more weight to fitting the conditional density of $y_{t}$ when $0<w<1$. For the special case where $w=1$, we obtain the classical pseudo-true parameter $\psi_{0}^{*}(1) \in \Psi$ of the ML estimator that minimizes the KL divergence. The proofs of all theorems presented in this section can be found in Appendix B.

Proposition 1 below states well known conditions for the strict stationarity and ergodicity (SE) of the true processes $\left\{f_{\tau}^{m}\right\}_{\tau \in \mathbb{Z}},\left\{x_{\tau}^{m}\right\}_{\tau \in \mathbb{Z}}$ and $\left\{y_{t}\right\}_{t \in \mathbb{Z}}$ generated by the linear Gaussian system in (11), (12) and (13) initialized in the infinite past.

Proposition 1. Let $\left\{x_{\tau}^{m}\right\}_{\tau \in \mathbb{Z}}$ and $\left\{y_{t}\right\}_{t \in \mathbb{Z}}$ be generated according to (11), (12) and (13) 
with

(i) $\left\|T_{f}\right\|<1,0<\left\|R_{f}\right\|<\infty$ and $0<\left\|\Sigma_{\eta}^{2}\right\|<\infty$ in (11);

(ii) $\left|\beta_{x}\right|<\infty$ and $0<\sigma_{\epsilon}^{2}<\infty$ in (12);

(iii) $\left|\beta_{y}\right|<\infty$ and $0<\sigma_{\zeta}^{2}<\infty$ in (13).

Then $\left\{x_{\tau}^{m}\right\}_{\tau \in \mathbb{Z}}$ and $\left\{y_{t}\right\}_{t \in \mathbb{Z}}$ are SE sequences with bounded moments of any order; i.e. $\mathbb{E}\left|x_{\tau}^{m}\right|^{r}<\infty$ and $\mathbb{E}\left|y_{t}\right|^{r}<\infty \forall r>0$.

Theorem 1 ensures the existence of the WML estimator as a random variable that takes values in the arg max set of the random likelihood function.

Theorem 1. (Existence) For given $w \in[0,1]$, let $(\Psi, \mathfrak{B}(\Psi))$ be a compact measurable space. Then there exists a.s. a measurable map $\hat{\psi}_{T}\left(w, \tilde{f}_{1}^{m}\right): \Omega \rightarrow \Psi$ satisfying

$$
\hat{\psi}_{T}\left(w, \tilde{f}_{1}^{m}\right) \in \arg \max _{\psi \in \Psi} \mathcal{L}_{T}\left(\psi, w, \tilde{f}_{1}^{m}\right),
$$

for all $T \in \mathbb{N}$ and every filter initialization $\tilde{f}_{1}^{m}$.

Theorem 2 establishes the strong consistency of the WML estimator of the true parameter vector $\psi_{0} \in \Psi$ for any choice of weight $w \in(0,1]$ for the likelihood. This result is obtained under the assumption that the mixed frequencies common factor model is well-specified and for any filter that identifies the parameter vector $\psi_{0} \in \Psi$ and is asymptotically SE with bounded moments of second order. The identification conditions and exponential almost sure (e.a.s.) convergence of different filters to an SE process with bounded second moment is well known and easy to establish in this linear Gaussian setting. For this reason, we do not repeat them here; see e.g. Mehra (1970) for such results on the classical Kalman filter, Bougerol (1993) for extensions, and Blasques, Koopman, and Lucas (2014) for identification, convergence results and bounded moments on a wide range of observation-driven filters. Theorem 2 thus assumes that $\psi_{0}$ maximizes the likelihood and assumes the convergence of the filtered sequence $\left\{\tilde{f}_{\tau}^{m}\left(\tilde{f}_{1}^{m}\right)\right\}_{\tau \in \mathbb{N}}$ initialized at $\tilde{f}_{1}^{m}$ to a unique limit SE sequence $\left\{\tilde{f}_{\tau}^{m}\right\}_{\tau \in \mathbb{Z}}$ with bounded second moment. Notice that we just require identification in the usual ML setting $w=1$; i.e. identification w.r.t. the unweighted likelihood function $\mathcal{L}_{T}(\psi, 1)$. As shown in the proof, identification of $\psi_{0}$ in $\mathcal{L}_{T}(\psi, 1)$ implies identification of $\psi_{0}$ in $\mathcal{L}_{T}(\psi, w)$ for any $w \in(0,1]$.

Theorem 2. (Consistency) Let $\left\{x_{\tau}^{m}\right\}$ and $\left\{y_{t}\right\}$ be generated by the mixed frequencies common factor model defined in (11), (12) and (13) under some $\psi_{0} \in \Psi$, and suppose that the conditions of Propositions 1 and Theorem 1 hold. Suppose furthermore that

$$
\mathcal{L}_{\infty}\left(\psi_{0}, 1\right)>\mathcal{L}_{\infty}(\psi, 1) \forall \psi \neq \psi_{0}
$$

and there exists a unique SE sequence such that

$$
\left\|\tilde{f}_{\tau}^{m}\left(\tilde{f}_{1}^{m}\right)-\tilde{f}_{\tau}^{m}\right\| \stackrel{\text { e.a.s. }}{\rightarrow} 0 \forall \tilde{f}_{1}^{m} \quad \text { as } \quad \tau \rightarrow \infty \quad \text { with } \quad \mathbb{E}\left|\tilde{f}_{\tau}^{m}\right|^{2}<\infty .
$$


Then the WML estimator $\hat{\psi}_{T}\left(w, \tilde{f}_{1}^{m}\right)$ satisfies

$$
\hat{\psi}_{T}\left(w, \tilde{f}_{1}^{m}\right) \stackrel{a . s .}{\rightarrow} \psi_{0} \quad \text { as } T \rightarrow \infty
$$

for any choice of weight $w \in(0,1]$ and any initialization $\tilde{f}_{1}^{m}$.

If the data $\left\{x_{\tau}^{m}\right\}$ and $\left\{y_{t}\right\}$ are obtained from an unknown data generating process but satisfy some regularity conditions, then we can still prove consistency of the WML estimator to pseudo-true parameter $\psi_{0}^{*}(w) \in \Psi$ that depends on the choice of weight $w \in(0,1]$.

It is well known that the classical ML estimator converges to a limit pseudo-true parameter that minimizes the KL divergence between the true joint probability measure of the data and the measure implied by the model. Theorem 3 characterizes the limit pseudo-true parameter $\psi_{0}^{*}(w)$ as the minimizer of a transformed KL divergence for every given $w \in(0,1]$. Just like the KL divergence, this new transformed divergence is also a pre-metric on the space of probability measures. The transformed KL divergence is further shown to be a weighted average of two KL divergences that is bounded from above (for $w=1$ ) by the KL divergence of the joint density of $y_{t}$ and $x_{\tau}$, and bounded from below (for $w=0$ ) by the conditional density of $y_{t}$ given $x_{\tau}^{m}$. For $w \in(0,1)$ the WML estimator converges to a pseudo-true parameter that gives more weight to the fit of the conditional model for $y_{t}$ than the standard ML estimator.

Below we let $p$ denote the true joint density of the vector $z_{t}:=\left(y_{t}, x_{t}^{\prime}\right)^{\prime}$, where $x_{t}$ is the stacked vector of monthly variables $x_{\tau}^{m}$, and let $p\left(z_{t}\right)=p_{1}\left(y_{t} \mid x_{t}\right) \cdot p_{2}\left(x_{t}\right)$ so that $p_{1}$ denotes the true conditional density and $y_{t}$ given $x_{t}$ and $p_{2}$ the true marginal of $x_{t}$. Similarly, we let $q(\cdot ; \psi)$ denote the joint density of $z_{t}$ as defined by our parametric model under $\psi \in \Psi$, and let $q_{1}(\cdot ; \psi)$ and $q_{2}(\cdot ; \psi)$ be the counterparts of $p_{1}$ and $p_{2}$ for the parametric model density. Finally, given any two densities $a$ and $b$, we let $\operatorname{KL}(a, b)$ denote the KL divergence between $a$ and $b$.

Theorem 3. (Consistency) Let $\left\{x_{\tau}^{m}\right\}$ and $\left\{y_{t}\right\}$ be SE and satisfy $\mathbb{E}\left|x_{\tau}^{m}\right|^{2}<\infty$ and $\mathbb{E}\left|y_{t}\right|^{2}<$ $\infty$. Furthermore, let the conditions of Theorem 1 hold and suppose that

$$
\mathcal{L}_{\infty}\left(\psi_{0}^{*}(w), w\right)>\mathcal{L}_{\infty}(\psi, w) \forall \psi \neq \psi_{0}^{*}(w)
$$

and there exists a unique SE sequence such that

$$
\left\|\tilde{f}_{\tau}\left(\tilde{f}_{1}^{m}\right)-\tilde{f}_{\tau}\right\| \stackrel{\text { e.a.s. }}{\rightarrow} 0 \forall \tilde{f}_{1}^{m} \text { as } \tau \rightarrow \infty \quad \text { with } \quad \mathbb{E}\left|\tilde{f}_{\tau}\right|^{2}<\infty .
$$

Then

$$
\hat{\psi}_{T}\left(w, \tilde{f}_{1}^{m}\right) \stackrel{\text { a.s. }}{\rightarrow} \psi_{0}^{*}(w) \text { as } T \rightarrow \infty
$$

for any initialization $\tilde{f}_{1}^{m}$ and any weight $w \in(0,1]$. Furthermore, the pseudo-true param- 
eter $\psi_{0}^{*}(w)$ minimizes a transformed $K L$ divergence

$$
\operatorname{TKL}_{w}(q(\cdot ; \psi), p)=\operatorname{KL}\left(q_{1}(\cdot ; \psi), p_{1}\right)+w \mathrm{KL}\left(q_{2}(\cdot ; \psi), p_{2}\right)
$$

which is a pre-metric on the space of distributions satisfying for any $w \in(0,1]$,

$$
\begin{gathered}
\operatorname{TKL}_{1}(q(\cdot ; \psi), p)=\operatorname{KL}(q(\cdot ; \psi), p) \quad, \quad \operatorname{TKL}_{0}(q(\cdot ; \psi), p)=\mathrm{KL}\left(q_{1}(\cdot ; \psi), p_{1}\right), \\
\operatorname{KL}\left(q_{1}(\cdot ; \psi), p_{1}\right) \leq \operatorname{TKL}(q(\cdot ; \psi), p) \leq \mathrm{KL}(q(\cdot ; \psi), p), \\
\text { and } \quad \operatorname{TKL}(q(\cdot ; \psi), p)=0 \quad \text { if and only if } \quad \operatorname{KL}\left(q_{1}(\cdot ; \psi), p_{1}\right)=0 .
\end{gathered}
$$

Theorem 4 establishes the asymptotic normality of the WML estimator under the assumption that the mixed frequencies dynamic factor model is well specified. Below we let $\mathcal{J}\left(\psi_{0}, w\right):=\mathbb{E} \ell_{t}^{\prime}\left(\psi_{0}, w\right) \ell_{t}^{\prime}\left(\psi_{0}, w\right)^{\top}$ denote the expected outer product of gradients and $\mathcal{I}\left(\psi_{0}, w\right):=\mathbb{E} \ell_{t}^{\prime \prime}\left(\psi_{0}, w\right)$ be the Fisher information matrix. The asymptotic normality proof is written for filters whose derivative processes are asymptotically SE and have bounded moments; see Blasques et at. (2014) for a wide range of observation-driven filters satisfying such conditions. Below, $\left\{\tilde{d} f_{\tau}^{m}\left(\tilde{d} f_{1}^{m}\right)\right\}$ and $\left\{\tilde{d} \tilde{d} f_{\tau}^{m}\left(\tilde{d} \tilde{d}_{1}^{m}\right)\right\}$ denote the first and second derivatives of the filter w.r.t. the parameter vector $\psi$, initialized at $\tilde{d f}_{1}^{m}$ and $\tilde{d d} f_{1}^{m}$ respectively. Their SE limits are denoted $\left\{\tilde{d} f_{\tau}^{m}\right\}$ and $\left\{\tilde{d} f_{\tau}^{m}\right\}$. Note that asymptotic normality result holds for any weight $w \in(0,1]$, but the asymptotic distribution of the WML estimator depends on the choice of weight $w$.

Theorem 4. (Asymptotic Normality) Let the conditions of Theorem 2 hold and $\psi_{0}$ be a point in the interior of $\Psi$. Suppose furthermore that there exists a unique SE sequence $\left\{\tilde{d} f_{\tau}^{m}\right\}$ such that

$$
\left\|\tilde{d} f_{\tau}^{m}\left(\tilde{d} f_{1}^{m}\right)-\tilde{d f} f_{\tau}^{m}\right\| \stackrel{\text { e.a.s. }}{\rightarrow} 0 \forall \tilde{d f}_{1}^{m} \quad \text { as } \quad \tau \rightarrow \infty \quad \text { with } \quad \mathbb{E}\left|\tilde{d} f_{\tau}^{m}\right|^{4}<\infty
$$

and a unique SE sequence $\left\{\tilde{d} \tilde{d} f_{\tau}\right\}$ such that

$$
\left\|\tilde{d d} f_{\tau}^{m}\left(\tilde{d} f_{1}^{m}\right)-\tilde{d} f_{\tau}^{m}\right\| \stackrel{\text { e.a.s. }}{\rightarrow} 0 \forall \tilde{d} \tilde{d} f_{1}^{m} \quad \text { as } \quad \tau \rightarrow \infty \quad \text { with } \quad \mathbb{E}\left|d \tilde{d} f_{\tau}^{m}\right|^{2}<\infty .
$$

Then, for every $\tilde{f}_{1}^{m}$ and every $w \in(0,1]$, the $M L$ estimator $\hat{\psi}_{T}\left(\tilde{f}_{1}^{m}\right)$ satisfies

$$
\sqrt{T}\left(\hat{\psi}_{T}\left(\tilde{f}_{1}^{m}, w\right)-\psi_{0}\right) \stackrel{d}{\rightarrow} N\left(0, \mathcal{I}^{-1}\left(\psi_{0}, w\right) \mathcal{J}\left(\psi_{0}, w\right) \mathcal{I}^{-1}\left(\psi_{0}, w\right)\right) \text { as } T \rightarrow \infty .
$$

Naturally, we can extend the asymptotic normality results to the mis-specified mixed measurement dynamic factor model by centering the WML estimator at the pseudo-true parameter $\psi_{0}^{*}(w)$.

Theorem 5. (Asymptotic Normality) Let the conditions of Theorem 3 hold and $\psi_{0}^{*}(w)$ be a point in the interior of $\Psi$. Suppose further that $\left\{x_{\tau}^{m}\right\}$ and $\left\{y_{t}\right\}$ are SE and satisfy 
$\mathbb{E}\left|x_{\tau}^{m}\right|^{4}<\infty$ and $\mathbb{E}\left|y_{t}\right|^{4}<\infty$ and there exists a unique SE sequence $\left\{\tilde{d} f_{\tau}^{m}\right\}$ such that

$$
\left\|\tilde{d} f_{\tau}^{m}\left(\tilde{d} f_{1}^{m}\right)-\tilde{d f} f_{\tau}^{m}\right\| \stackrel{\text { e.a.s. }}{\rightarrow} 0 \forall \tilde{d f}_{1}^{m} \quad \text { as } \quad \tau \rightarrow \infty \quad \text { with } \quad \mathbb{E}\left|\tilde{d} f_{\tau}^{m}\right|^{4}<\infty
$$

and a unique SE sequence $\left\{\tilde{d \tilde{d}} f_{\tau}\right\}$ such that

$$
\left\|\tilde{d} f_{\tau}^{m}\left(\tilde{d} f_{1}^{m}\right)-\tilde{d} \tilde{d} f_{\tau}^{m}\right\| \stackrel{e . a_{s} s .}{\rightarrow} 0 \forall d \tilde{d} f_{1}^{m} \quad \text { as } \quad \tau \rightarrow \infty \quad \text { with } \quad \mathbb{E}\left|d \tilde{d} f_{\tau}^{m}\right|^{2}<\infty .
$$

Then, for every $\tilde{f}_{1}^{m}$ and every $w \in(0,1]$, the $M L$ estimator $\hat{\psi}_{T}\left(w, \tilde{f}_{1}^{m}\right)$ satisfies

$\sqrt{T}\left(\hat{\psi}_{T}\left(\tilde{f}_{1}^{m}\right)-\psi_{0}^{*}(w)\right) \stackrel{d}{\rightarrow} N\left(0, \mathcal{I}^{-1}\left(\psi_{0}^{*}(w), w\right) \mathcal{J}\left(\psi_{0}^{*}(w), w\right) \mathcal{I}^{-1}\left(\psi_{0}^{*}(w), w\right)\right) \quad$ as $\quad T \rightarrow \infty$

\subsection{Small Sample Properties of WML: Monte Carlo Study}

Next we investigate the finite sample effects of different choices for the value of $W$ in (26) on the in-sample fit in different scenarios using Monte Carlo simulations. We generate data for $y_{t}$ and $x_{\tau}^{m}$ for different number of variables, $k=2, k=5$ and $k=10$. The length of the time series is set to $n=100$ for all scenarios. We consider two different data generating processes (DGPs) for the vector of observations $z_{t}=\left(y_{t}, x_{t}^{\prime}\right)^{\prime}$ in the simulations, where $x_{t}$ is the stacked vector of monthly variables $x_{\tau}^{m}$.

The first DGP for $z_{t}$ is a multivariate common factor model with one common factor $f_{\tau}$ and one idiosyncratic factor $u_{\tau}^{(i)}$ for each variable. The model is given by

$$
z_{t}=\beta_{z} f_{t}+u_{t}+\varepsilon_{t}, \quad \varepsilon_{\tau} \sim N I D\left(0, \sigma_{\varepsilon}^{2}\right)
$$

All factors, both common and idiosyncratic, are driven by AR processes of order 1 as in (19) and the autoregressive parameters are all set at 0.80 . The factor loadings of variable $x^{(i)}$ on the common factor are set equal to $\frac{1}{i}$, for $i=1, \ldots, k$. Furthermore, we choose $\sigma_{\varepsilon}=0.50$ and $\sigma_{\eta}=0.25$.

The second DGP for $z_{t}$ is the multivariate $\operatorname{VAR}(1)$ process

$$
z_{t}=\Phi z_{t-1}+\varepsilon_{t}, \quad \varepsilon_{\tau} \sim N I D\left(0, \sigma_{\varepsilon}^{2}\right)
$$

where all diagonal elements of the $k \times k$ matrix $\Phi$ are set equal to 0.80 and the offdiagonal elements are randomly generated values between -0.5 and 0.5 but we only allow for stationary VAR processes. As in the first DGP, we choose $\sigma_{\varepsilon}=0.50$. In our simulation study, we consider three different scenarios in particular.

Scenario 1: Underspecification In the first scenario, we adopt the common dynamic factor model (27) as the DGP but for the analysis we consider the model

$$
z_{t}=\beta_{z} f_{t}+\varepsilon_{t}, \quad \varepsilon_{\tau} \sim N I D\left(0, \sigma_{\varepsilon}^{2}\right)
$$

This model does not include idiosyncratic factors and hence it is underspecified. 
We expect that in this setting we can improve the in-sample accuracy for $y_{t}$ when we take the value of $W$ larger than unity. For different dimensions, $k=2, k=5$ and $k=10$, we generate 1,000 datasets from the first DPG and we estimate the parameters from the model (29) with values of $W$ ranging from 1 to 25.

Scenario 2: Misspecification In the second scenario, we adopt the VAR(1) model (28) as DGP. Similarly as in the first scenario, we generate 1,000 datasets from the $\operatorname{VAR}(1)$ with $k=2, k=5$ and $k=10$, and we estimate the parameters of the dynamic factor model (29) with values of $W$ ranging from 1 to 25 . Since the DGP model is different from the model that we consider for te analysis, the model is misspecified. Hence we expect that increasing the value of $W$ can be beneficial for the in-sample fit of $y_{t}$. Theorem 3 has shown that such large improvements are explained by the fact that we can use the weight $W$ to control the limit pseudotrue parameter to which the weighted MLE will converge. In particular, the larger the $W$, the more the WML estimator will focus on minimizing the KL divergence between the true conditional density of $y_{t}$ and the conditional density implied by the model.

Scenario 3: Correct Specification In the third and final scenario, we adopt the dynamic factor (27) as DGP and we consider the same model in the analysis. We generate 1,000 datasets for $k=2, k=5$ and $k=10$, and we estimate the parameters for values of $W$ ranging from 1 to 25 . This model is correctly specified and we expect that increasing the value of $W$ will not improve the in-sample fit for $y_{t}$ much in comparison to the previous two scenarios. In effect, in Theorem 2 we have shown that asymptotically the different values of $W$ must yield the same results since the weighted MLE is consistent to the true parameter for any $W$. Any improvements in the correct specification setting are thus only finite-sample improvements.

In the left panel of Table 2 we present the mean squared error (MSE) averages for the variable of interest $y_{t}$. Each column is scaled to the value at $W=1$, the maximum likelihood estimate. From Table 2 we find that increasing the value of $W$ leads to a better in-sample fit for $y_{t}$ for all three dimensions $k$. However it is not necessary to choose very large values for $W$. The improvements in MSE appear to converge to some upper limit for increasing values of $W$. To illustrate we also report the results for $W=1,000$ from which we learn that there is not much difference with $W=25$. Furthermore, we observe that more gains can be made when more variables are included in the model.

In the first panel of Figure 1 we present the MSE of $y_{t}$ and the average MSEs for the variables of $x_{t}$ for $W=1, \ldots, 25$. As we have learned from Table 2 , increasing the value of $W$ improves the in-sample fit for $y_{t}$ while the actual improvements diminish when $W$ increases. It comes at the cost of the accuracy for the variables in $x_{t}$. The right panel of each figure presents the average MSE for $x_{t}$ and it is clear that increasing $W$ leads to a worse in-sample fit for these variables. We also find that the marginal effect becomes smaller for larger values of $W$. Since the MSE of $y_{t}$ is decreasing as a function of $W$, 
there is no clear optimal value for $W$. The choice of $W$ should depend on how important the different variables are considered to be.

For scenario 2 we present the improvements in average MSE for $y_{t}$ in the middle panel of Table 2. As in the first scenario, we observe that increasing the value of $W$ leads to a better in-sample fit for $y_{t}$ for all the three values of $k$, and again the improvement in MSE seems to converge to some upper limit for increasing values of $W$. This is consistent with the findings of Theorem 3. We can also conclude that the gains for $k=5$ and $k=10$ are much larger than in scenario 1 . However, more gains can be made in the model with $k=5$ than in the model with $k=10$.

Table 2: Average MSE of target variable $y_{t}$ for different values of $W$

\begin{tabular}{|c|c|c|c|c|c|c|c|c|c|}
\hline \multirow[b]{2}{*}{ W } & \multicolumn{3}{|c|}{ Scenario 1} & \multicolumn{3}{|c|}{ Scenario 2} & \multicolumn{3}{|c|}{ Scenario 3} \\
\hline & $k=2$ & $k=5$ & $k=10$ & $k=2$ & $k=5$ & $k=10$ & $k=2$ & $k=5$ & $k=10$ \\
\hline 1 & 1.000 & 1.000 & 1.000 & 1.000 & 1.000 & 1.000 & 1.0000 & 1.0000 & 1.0000 \\
\hline 2 & 0.983 & 0.962 & 0.931 & 0.977 & 0.890 & 0.952 & 0.9996 & 0.9994 & 0.9999 \\
\hline 3 & 0.974 & 0.947 & 0.889 & 0.973 & 0.737 & 0.891 & 0.9994 & 0.9994 & 0.9998 \\
\hline 4 & 0.972 & 0.942 & 0.874 & 0.973 & 0.653 & 0.834 & 0.9993 & 0.9993 & 0.9997 \\
\hline 5 & 0.970 & 0.938 & 0.865 & 0.973 & 0.592 & 0.812 & 0.9992 & 0.9993 & 0.9993 \\
\hline 10 & 0.968 & 0.928 & 0.844 & 0.972 & 0.509 & 0.718 & 0.9990 & 0.9990 & 0.9989 \\
\hline 15 & 0.967 & 0.925 & 0.837 & 0.971 & 0.488 & 0.713 & 0.9989 & 0.9989 & 0.9986 \\
\hline 25 & 0.966 & 0.920 & 0.831 & 0.969 & 0.476 & 0.705 & 0.9988 & 0.9987 & 0.9984 \\
\hline 1000 & 0.965 & 0.914 & 0.809 & 0.965 & 0.442 & 0.685 & 0.9986 & 0.9976 & 0.9958 \\
\hline
\end{tabular}

This table presents the average MSE for the target variable $y_{t}$ over 1,000 simulation runs. In scenario 1 an underspecified DFM (without idiosyncratic factors) is estimated on a DFM with idiosyncratic factors. In scenario 2 a DFM (without idiosyncratic factors) is estimated on a dataset with a VAR(1) process as DGP and in scenario 3 a DFM model with idiosyncratic factors is estimated on a dataset with the same model as the DGP. For each column all numbers are scaled to the value at $W=1$.

Finally, for scenario 3 we present the results in the right panel of Table 2. The improvements in in-sample fit are negligible when the value of $W$ increases. This is consistent with Theorem 2, as any improvements can only be attributed to small-sample variations. 


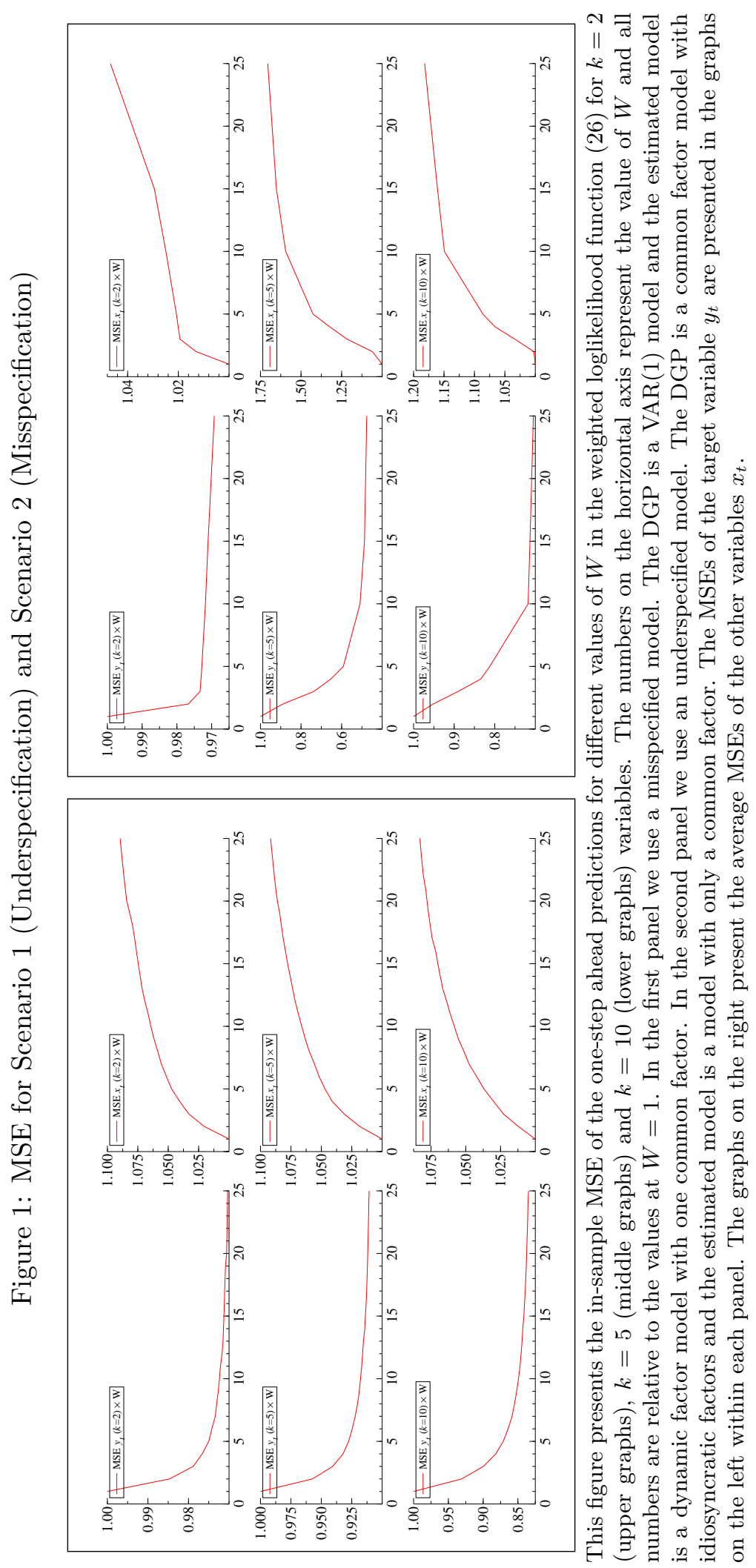




\section{Empirical Results}

In our empirical study we consider four different methods for treating mixed frequency dynamic factor models (DFMs): the MFI method of Section 3.2, the MFA method of Section 3.3 and the MFS-M and MFS-Q methods of Section 3.4. We first present the estimation results for the four models based on the original dataset that was used by Mariano and Murasawa (2003). We will evaluate the forecasting and nowcasting accuracy for the various methods and compare the results. These different approaches to handle mixed-frequencies with a dynamic factor model, are all examples of full system approaches. We will also compare the forecasting and nowcasting results with two partial model approaches: Bridge models of Trehan (1989) and the MIDAS regression of Ghysels, Santa-Clara, and Valkanov (2006). We refer for some details of these two approaches to the Technical Appendix. Furthermore we assess the improvements in forecasting and nowcasting accuracy when we adopt the method of weighted maximum likelihood that is presented in Section 4.

\subsection{DATA}

The original Mariano and Murasawa (2003) data set consist of quarterly observed US real GDP and four monthly observed indicators; see Table 3 for their descriptions. The sample period is January 1959 until December 2000. The first difference of natural log for each series is taken and multiplied by 100, which is approximately equal to the quarterly or monthly growth rate. The number of parameters in the model is reduced by demeaning all series, so that no intercept coefficients are required. Furthermore, the loading of the dynamic factor on quarterly GDP is fixed at a value of 1 for identification purposes. In the forecasting and nowcasting studies, we use an extended version of this dataset, with the sample period from January 1960 until December 2009.

Table 3: Data definitions

\begin{tabular}{ll}
\hline Indicator & Description \\
\hline GDP & Real GDP (billions of chained $1996 \$, \mathrm{SA}, \mathrm{AR})$ \\
& \multicolumn{1}{c}{ Monthly } \\
EMP & Employees on non-agricultural payrolls (thousands, SA) \\
INC & Personal income less transfer payments (billions of chained 1996 $\$, \mathrm{SA}, \mathrm{AR})$ \\
IIP & Index of industrial production (1992 = 100, SA) \\
SLS & Manufacturing and trade sales (millions of chained $\$, \mathrm{SA})$ \\
\hline
\end{tabular}

This table presents the definitions of all the quarterly and monthly variables that are used in the models in this section and the abbreviations that are used for these variables. SA means 'seasonally adjusted' and AR means 'annual rate'. 


\subsection{Parameter Estimates by Maximum Likelihood}

We have obtained our estimation results by following Mariano and Murasawa (2003) closely. We have set the variances of the disturbance terms in the observation equations of all models equal to zero and we also adopt the orders that they use for the $\operatorname{AR}(p)$ processes by which the factors are modeled. That is, the common factor $f_{t}$ is modeled by an $\mathrm{AR}(1)$ process and all idiosyncratic factors $u_{t}$ and $v_{t}$ are modeled by $\mathrm{AR}(2)$ processes for all models.

The parameter estimates for MFI model are presented in the first panel of Table 4 . These estimates are very similar to the estimates obtained by Mariano and Murasawa (2003) for the same dataset. The MFS-M model targets the same modeling framework as assumed for the MFI model. However, the MFI model describes the dynamic information for $\Delta$ ln GDP from a high frequency monthly process, while the MFS-M model adopts a low frequency quarterly dynamic process. Overall, we obtain similar estimation results for both methods. The only notable difference is in the estimated parameters $\phi_{u, 1}$ and $\phi_{u, 2}$ for $\Delta$ ln GDP. The third panel presents the parameter estimates for the MFS-Q model, which are also very similar to the estimates of the MFI and MFS-M methods. However, we notice that all elements of $\beta_{x}$ are now estimated at about $\frac{1}{3}$ of their value in the MFI and MFS-M models: in the MFS-Q model this is the loading on the quarterly value of $f_{t}$, whereas in the MFI and MFS-M models it is the loading on the monthly value of $f_{t}$. Finally, the fourth panel reports the parameter estimates for the MFA model. Given that the number of observations for the quarterly model has reduced, the variances of the idiosyncratic factors have increased for all four indicators. The estimates of the other variables are quite similar to those of the MFS-M model, with the exception of the AR process for $\Delta \ln \operatorname{EMP}\left(x^{(1)}\right)$. This process has become rather persistent with an estimated $\phi_{1,1}$ of 0.69 when $\Delta$ ln EMP is modeled by a quarterly frequency. When it is modeled with a monthly frequency, the estimate of $\phi_{1,1}$ has been close to zero, while the $\phi_{1,2}$ estimates have been at 0.45 and 0.43 in the MFI and MFS-M models, respectively.

\subsection{Nowcasting and Forecasting Comparisons}

Next we compare the forecasting results of our three approaches at handling mixedfrequencies in the dynamic factor model. We also compare these results to the forecasting results that are obtained with bridge models and MIDAS regressions.

We compare the models using the same set of variables as in Mariano and Murasawa (2003), but with the data extended until December 2009. Table 5 presents the MSE of the predictions for the quarterly observed $y_{t}$ in the different models with forecasting horizons $h=0,1,2,3,6$ months ahead. These predictions are made using a rolling window of 25 years of data to estimate the parameters. Forecasting horizons of $h=0,1,2$ are usually referred to as nowcasting. When $h=0$, all values of $x_{t}$ are known until the quarter that is to be forecasted. When $h=1$, the values of $x_{t}$ are known until the first two months of the quarter. When $h=2$, only the first month of the quarter that we 


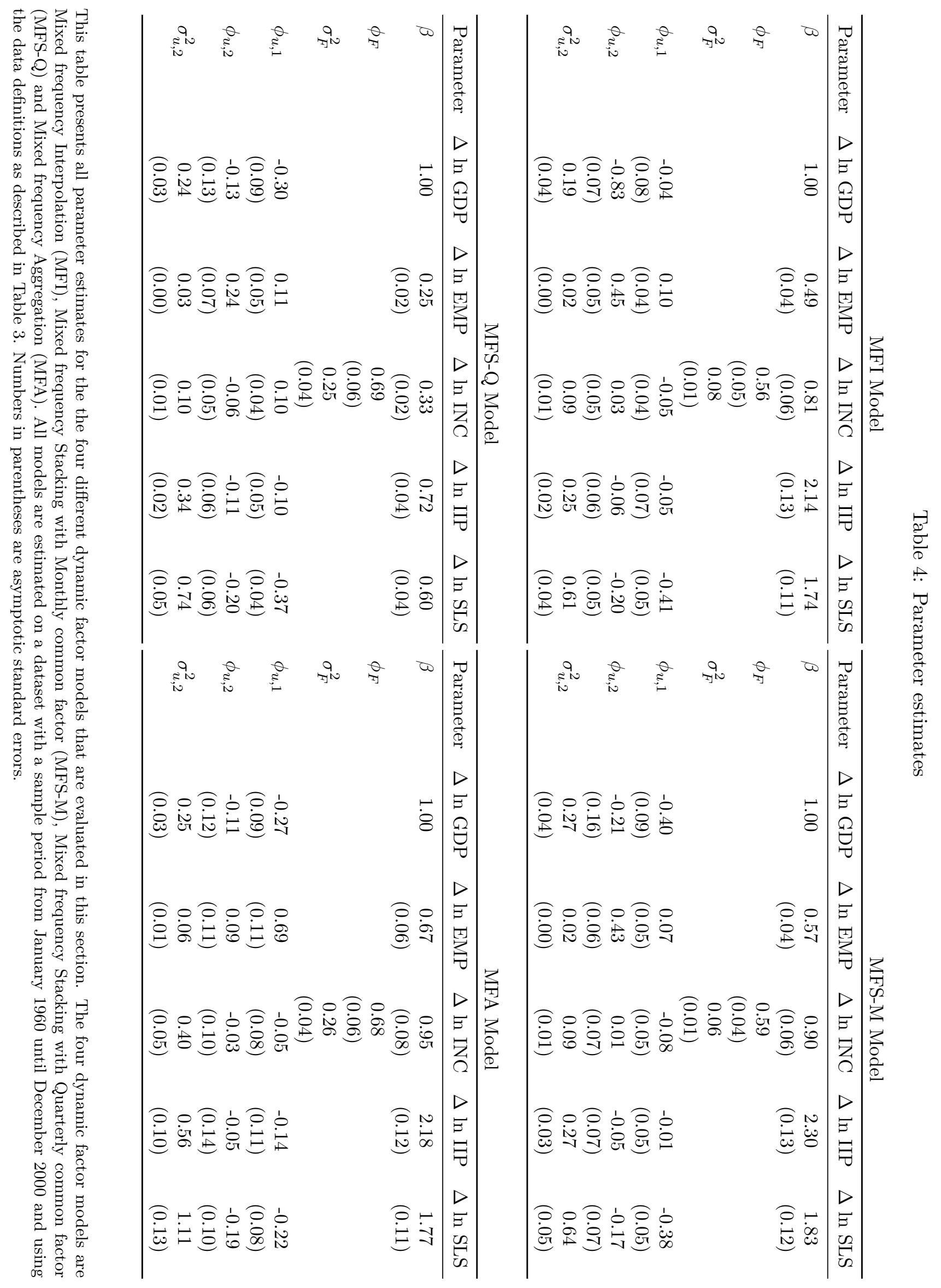


want to forecast is observed. When $h=3$, we are forecasting one quarter ahead, no observations are available for the quarter that we forecast. All values until the previous quarter are observed. Similarly, when $h=6$ we are forecasting two quarters ahead without observations being available from the quarter to forecast and its previous quarter. In the MFA model, there are no monthly dynamics, so it is only possible to forecast at $h=0,3,6$.

From Table 3 we learn that the MFS-M model provides the more accurate $\Delta \ln$ GDP predictions for all forecasting horizons. The accuracy is higher than the original MFI model. The MFA model is only more accurate for $h=3$ and $h=6$ while the MFS-Q model is most accurate for $h=6$ while it is less accurate than both the MFI and MFS-M models for all other horizons.

When we further take into account the benchmark Bridge and MIDAS models, we find that the predictions of the MIDAS model are most accurate for nowcasting at $h=0$ and $h=1$. For longer forecasting horizons, MIDAS forecasts become less accurate.

We consult the Diebold and Mariano (1995) test to verify whether forecast accuracy differences are significant at the $5 \%$ level. In our study, the only significant differences between any of the models for the nowcasting horizons $h=0,1,2$ is found for (i) $h=2$, where the MFS-M model is more accurate than the MFS-Q model; (ii) $h=3$, where the MFA model is significantly more accurate than the MFI and MIDAS models; (iii) $h=6$, where the MFS-Q model is significantly more accurate than all other models and the MFA model is significantly more accurate than the rest of the models, except for the MFS-M model. Given the large similarity in the structure of these models and their parameter estimates, it is perhaps only surprising that we find any difference at all. Finally, we have found that the MIDAS model performs significantly worse than all of the other models for $h=6$.

Table 5: Forecast comparisons for US GDP growth

\begin{tabular}{lccccc}
\hline & $h=0$ & $h=1$ & $h=2$ & $h=3$ & $h=6$ \\
\hline MFI & 0.1779 & 0.1918 & 0.2340 & 0.3156 & 0.4023 \\
MFS-M & 0.1666 & 0.1730 & 0.2108 & 0.2935 & 0.3986 \\
MFS-Q & 0.1765 & 0.1909 & 0.2411 & 0.2989 & 0.3701 \\
MFA & 0.1693 & & & 0.2809 & 0.3754 \\
BM & 0.1833 & 0.2056 & 0.2455 & 0.3046 & 0.4180 \\
MIDAS & 0.1597 & 0.1658 & 0.2464 & 0.3635 & 0.4873 \\
\hline
\end{tabular}

This table presents the forecast MSEs for the quarterly observed US GDP growth from January 2000 until December 2009 for four dynamic factor models and two benchmark models at forecasting horizons $h=$ $0,1,2,3,6$. The four dynamic factor models are Mixed frequency Interpolation (MFI), Mixed frequency Stacking with Monthly common factor (MFS-M), Mixed frequency Stacking with Quarterly common factor (MFS-Q) and Mixed frequency Aggregation (MFA). Benchmark models are Bridge models (BM) and MIDAS regressions. All results are based on parameter estimates obtained from the same dataset with sample period from January 1960 until December 2009 and using the data definitions as described in Table 3. For each forecasting horizon the MSEs of the two most accurate models are highlighted, where the darkest shade is used for the most accurate model. 


\subsection{Parameter Estimates by Weighted Maximum Likelihood}

Dynamic factor models are often considered in the simultaneous modeling of a group of variables, but all variables in the model are of equal importance to the likelihood function. However, it can be desirable to have somewhat more focus on fitting a particular variable of interest. In our case, this may be $\Delta \ln$ GDP. In Section 4 we have proposed a method to implement this idea by giving extra weight to the most important variables in the loglikelihood function. In our model we will give extra weight to GDP growth, the variable $y_{t}$.

How much additive weight we give to $y_{t}$ depends on the choice of $W$ in (26). To investigate the effects of different values of $W$ we study the in-sample fit. The MSE of the one-step ahead prediction errors of the Kalman filter is plotted as a function of $W$ for $y_{t}$ and for the four different series of $x_{t}$ in Figure 2 for the MFS-M model. For each variable, all MSE values are divided by the MSE value at $W=1$, in order to make the graphs comparable with each other.

By increasing $W$, the in-sample fit for $y_{t}$ improves considerably, but the in-sample fits for all $x_{t}$ become less accurate. Furthermore, from the first graph in Figure 2 it is clear that the marginal effect of increasing $W$ becomes less for higher values of $W$. For example, increasing $W$ from 1 to 6 leads to a $9.5 \%$ improvement in MSE for $y_{t}$ while increasing $W$ further to a value of 60 leads to a $14.0 \%$ improvement compared to $W=1$. At $W=6$ the largest increase in MSE is found for $x^{(3)}$ with $5.3 \%$, while at $W=60$ we find a $17.6 \%$ increase in MSE for this variable. Since the MSE of $y_{t}$ is decreasing as a function of $W$, there is not one clear optimal value for $W$, but the optimum depends on how important the different variables are.

From the results presented in Figure 2 and from the results of our Monte Carlo study in Section 4.2, we may conclude that choosing the optimal value for $W$ is a trade off between the gain in in-sample fit for $y_{t}$ and its loss for $x_{t}$. However, when we focus on the effect of $W$ on forecasting accuracy we obtain a different picture. In Table 6 we show the forecasting accuracy at different horizons for $y_{t}$ for different values of $W$ for the MFS-M model. It becomes apparent that increasing $W$ also improves the accuracy of the forecasts for all horizons. Furthermore, we find that increasing the weight too much eventually leads to a decrease in the forecasting accuracy, because the fit of the $x_{t}$ variables becomes worse with further increases in $W$. As a consequence, forecasted values for $x_{t+h}$ become less informative for the forecasts of $y_{t+h}$. In our study we find that $W=6$ is the optimal weight for $h=0,1,2$ and $W=5$ is optimal for $h=3$. For $h=6$ we find the lowest MSE with $W=4$, so the optimal value for $W$ appears to be lower for longer forecast horizons.

The Diebold and Mariano test indicates that the WML method with $W=6$ leads to a significant improvement at a $5 \%$ level over the model with $W=1$ (which provides the original estimates for the MFS-M model) for $h=0,1,2$ and at a $10 \%$ level for $h=3,6$. The parameter estimates with $W=5$ at $h=3$ and $W=4$ at $h=6$ are also significant improvements over the models with $W=1$ at these horizons. 
Figure 2: In-sample accuracy using WML
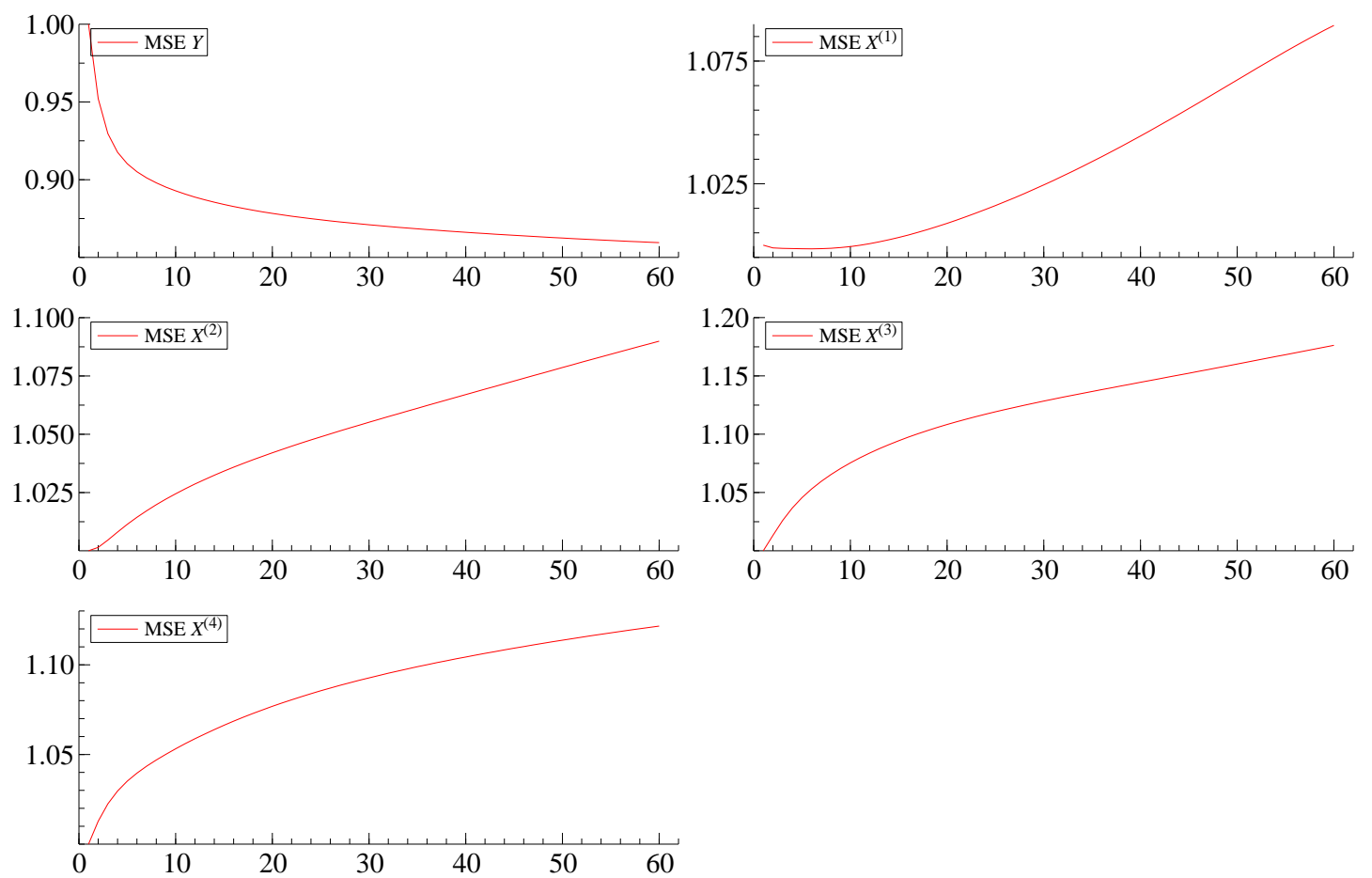

This figure presents the in-sample MSE of the one-step ahead predictions for all variables in the Mixed frequency Stacking model with Monthly common factor (MFS-M) for different values of $W$ in the weighted loglikelihood function (26). All models are estimated on the same dataset with sample period from January 1960 until December 2009 and using the data definitions as described in Table 3. The numbers on the horizontal axis represent the value of $W$ and all numbers are relative to the values at $W=1$.

In the next part of the study we take different integer values for $W$ to determine the optimal value of $W$ afterwards. Optimization techniques can be used to determine the optimal value of $W$ for each forecasting horizon, as $W$ is not limited to integer values only. However, our presented results provide a clear picture of the usefulness of the WML estimation method.

The weighted maximum likelihood method can be applied to all four dynamic factor models studied in this section. Based on our findings for the MFS-M model, we choose $W=6$ as the optimal value and we estimate the MFI, MFS-Q and MFA models. The forecasting accuracy of the models with $W=6$ is presented in Table 7 . We find that for all models and for all forecasting horizons we obtain clear improvements when compared with the results for the models with $W=1$ in Table 5 . These improvements are all significant at $5 \%$ level. Furthermore, the MFS-M model has produced the most accurate forecasts for all horizons. Only in the case of $h=6$ the MFI model performs best. For $h=2$ and higher, all four models perform better than the benchmark BM and MIDAS models. For these benchmark models, weighted maximum likelihood is not possible, because these models are estimated using least squares methods. 
Table 6: Forecast comparisons for US GDP growth with weighted likelihood function

\begin{tabular}{rccccc}
\hline $\mathrm{W}$ & $h=0$ & $h=1$ & $h=2$ & $h=3$ & $h=6$ \\
\hline 1 & 0.1666 & 0.1730 & 0.2108 & 0.2935 & 0.3917 \\
2 & 0.1600 & 0.1689 & 0.2049 & 0.2826 & 0.3708 \\
3 & 0.1571 & 0.1674 & 0.2028 & 0.2783 & 0.3614 \\
4 & 0.1556 & 0.1670 & 0.2013 & 0.2759 & 0.3534 \\
5 & 0.1517 & 0.1703 & 0.2004 & 0.2745 & 0.3662 \\
6 & 0.1513 & 0.1560 & 0.1914 & 0.2777 & 0.3733 \\
7 & 0.1611 & 0.1668 & 0.2034 & 0.2773 & 0.3715 \\
8 & 0.1608 & 0.1670 & 0.2033 & 0.2772 & 0.3699 \\
9 & 0.1612 & 0.1682 & 0.2032 & 0.2775 & 0.3683 \\
10 & 0.1614 & 0.1690 & 0.2033 & 0.2781 & 0.3662 \\
11 & 0.1615 & 0.1698 & 0.2035 & 0.2786 & 0.3577 \\
12 & 0.1617 & 0.1705 & 0.2037 & 0.2792 & 0.3572 \\
\hline
\end{tabular}

This table presents the MSEs of the forecasts for the quarterly observed US GDP growth for the Mixed frequency Stacking model with Monthly common factor (MFS-M) at forecasting horizons $h=0,1,2,3,6$ using different values for $W$ in the weighted loglikelihood function (26). All models are estimated on the same dataset with sample period from January 1960 until December 2009 and using the data definitions as described in Table 3. For each forecasting horizon the MSE of the most accurate model is highlighted.

Table 7: Forecast comparisons for US GDP growth with WML and $W=6$

\begin{tabular}{lccccc}
\hline & $h=0$ & $h=1$ & $h=2$ & $h=3$ & $h=6$ \\
\hline MFI & 0.1787 & 0.1885 & 0.2078 & 0.2841 & 0.3629 \\
MFS-M & 0.1513 & 0.1560 & 0.1914 & 0.2777 & 0.3733 \\
MFS-Q & 0.1630 & 0.1676 & 0.2249 & 0.2849 & 0.3670 \\
MFA & 0.1576 & & & 0.2809 & 0.3677 \\
BM & 0.1833 & 0.2056 & 0.2455 & 0.3046 & 0.4197 \\
MIDAS & 0.1597 & 0.1658 & 0.2464 & 0.3635 & 0.4873 \\
\hline
\end{tabular}

This table presents the MSEs of the forecasts for the quarterly observed US GDP growth for four different dynamic factor models and two benchmark models at forecasting horizons $h=0,1,2,3,6$ using $W=$ 6 in the weighted loglikelihood function (26) for the four dynamic factor models. The four dynamic factor models are Mixed frequency Interpolation (MFI), Mixed frequency Stacking with Monthly common factor (MFS-M), Mixed frequency Stacking with Quarterly common factor (MFS-Q) and Mixed frequency Aggregation (MFA). Benchmark models are Bridge models (BM) and MIDAS regressions. All models are estimated on the same dataset with sample period from January 1960 until December 2009 and using the data definitions as described in Table 3. For each forecasting horizon the MSEs of the two most accurate models are highlighted, where the darkest shade is used for the most accurate model.

However, the comparisons in Table 7 has given the MFS-M model some favor, because the value of $W=6$ was chosen based on this model. Therefore, we next determine the optimal (integer) value of $W$ for each model and for each forecasting horizon. These forecasting accuracy results are presented in Table 8. In the right panel of Table 8 we present the optimal values of $W$. The MFS-M model still performs best for all forecasting horizons, except for $h=6$. Furthermore, it is very difficult to detect a clear pattern in the optimal values of $W$. Therefore, when adopting this method in a practical setting for 
forecasting purposes, we would take a moderate fixed value for $W$, such as $W=5$.

Table 8: Forecast comparisons for US GDP growth with WML and optimal value for $W$

\begin{tabular}{lccccc}
\hline & \multicolumn{5}{c}{ Mean Square Error } \\
& $h=0$ & $h=1$ & $h=2$ & $h=3$ & $h=6$ \\
\hline MFI & 0.1687 & 0.1765 & 0.1966 & 0.2835 & 0.3559 \\
MFS-M & 0.1513 & 0.1560 & 0.1914 & 0.2745 & 0.3593 \\
MFS-Q & 0.1629 & 0.1670 & 0.2215 & 0.2835 & 0.3621 \\
MFA & 0.1576 & & & 0.2769 & 0.3566 \\
BM & 0.1833 & 0.2056 & 0.2455 & 0.3046 & 0.4197 \\
MIDAS & 0.1597 & 0.1658 & 0.2464 & 0.3635 & 0.4873 \\
\hline \multicolumn{7}{c}{ Optimal vale of $W$} \\
& $h=0$ & $h=1$ & $h=2$ & $h=3$ & $h=6$ \\
\hline MFI & 2 & 2 & 2 & 5 & 2 \\
MFS-M & 6 & 6 & 6 & 5 & 4 \\
MFS-Q & 7 & 8 & 3 & 3 & 2 \\
MFA & 6 & \multicolumn{5}{c}{2} & 8 \\
\hline
\end{tabular}

The first panel of this table presents the MSEs of the forecasts for the quarterly observed US GDP growth for four different dynamic factor models and two benchmark models at forecasting horizons $h=0,1,2,3,6$ using the optimal integer value for $W$ in the weighted loglikelihood function (26) for the four dynamic factor models. The optimal values for $W$ are presented in the second panel of the table. The four dynamic factor models are Mixed frequency Interpolation (MFI), Mixed frequency Stacking with Monthly common factor (MFS-M), Mixed frequency Stacking with Quarterly common factor (MFS-Q) and Mixed frequency Aggregation (MFA). Benchmark models are Bridge models (BM) and MIDAS regressions. All models are estimated on the same dataset with sample period from January 1960 until December 2009 and using the data definitions as described in Table 3. For each forecasting horizon the MSEs of the two most accurate models are highlighted, where the darkest shade is used for the most accurate model.

\section{Conclusions}

We have proposed solutions for two different problems that are often encountered in analyzing panels of economic and financial time series with different sample frequencies. The first problem is how to jointly model, say, monthly and quarterly time series. The default solution is to model both series as monthly variables and to allow for missing observations in the intermediate months for the quarterly variables. In our solution we opt for the modeling of the series in the lower quarterly sampling frequency and represent the monthly variable as a vector process to formulate the monthly dynamic process. There is no loss of high frequency information in our low frequency solution. We also show that in many situations, the low frequency solution leads to computational gains when compared to the high frequency solution. The second problem is how to focus on a set of key variables that we want to accurately forecast, in a simultaneous model. The typical setting is a dynamic factor analysis for a panel of time series with the purpose to forecast a small selection of variables in the panel. In our illustration it is a single series, the percentage growth in gross domestic product. We sacrifice some degree of fit for all other 
variables with the aim to improve the fit of the variable of interest. Our solution consists of adding more weight to the likelihood contribution by the variable of interest in the construction of the likelihood function for the joint model. The resulting weighted likelihood function turns out to be rather effective in the accomplishment of targeting a specific variable of interest, whether it is for improving in-sample or out-of-sample performance of the model. We also show that the parameter estimates obtained from the maximization of the weighted likelihood are consistent and asymptotically normally distributed. Their small sample properties are investigated under different settings in a Monte Carlo study. The presented illustration shows that our solutions lead to empirically relevant improvements in nowcasting and forecasting. We expect that our proposed solutions also have consequences in other applications and in other modeling frameworks. Further research in these directions are planned in future.

\section{A Derivations for Univariate Autoregressive Processes}

\section{A.1 AR(1) PROCESS With STACKED OBSERVATIONS}

Consider the AR(1) process of the monthly $(m)$ observed variable $x_{\tau}^{m}$ with monthly time index $\tau$

$$
x_{\tau}^{m}=\phi x_{\tau-1}^{m}+\varepsilon_{\tau}, \quad \varepsilon_{\tau} \sim N I D\left(0, \sigma_{\varepsilon}^{2}\right)
$$

When the monthly observations of $x_{\tau}^{m}$ are stacked into the quarterly $(q) 3 \times 1$ vectors $x_{t}^{q}$ with quarterly time index $t$, then the equations of the $\operatorname{AR}(1)$ process for the stacked observations can be written as

$$
x_{t, 1}^{q}=\phi x_{t-1,3}^{q}+\varepsilon_{t, 1}, \quad x_{t, 2}^{q}=\phi x_{t, 1}^{q}+\varepsilon_{t, 2}, \quad x_{t, 3}^{q}=\phi x_{t, 2}^{q}+\varepsilon_{t, 3}
$$

To develop a low frequency recursion for $x_{t}^{q}$, we substitute the first equation for the value of $x_{t, 1}^{q}$ and the second equation for $x_{t, 2}^{q}$ gives the following set of equations

$$
\begin{aligned}
x_{t, 1}^{q} & =\phi x_{t-1,3}^{q}+\varepsilon_{t, 1} \\
x_{t, 2}^{q} & =\phi\left(\phi x_{t-1,3}^{q}+\varepsilon_{t, 1}\right)+\varepsilon_{t, 2} \\
& =\phi^{2} x_{t-1,3}^{q}+\phi \varepsilon_{t, 1}+\varepsilon_{t, 2} \\
x_{t, 3}^{q} & =\phi\left(\phi^{2} x_{t-1,3}^{q}+\phi \varepsilon_{t, 1}+\varepsilon_{t, 2}\right)+\varepsilon_{t, 3} \\
& =\phi^{3} x_{t-1,3}^{q}+\phi^{2} \varepsilon_{t, 1}+\phi \varepsilon_{t, 2}+\varepsilon_{t, 3}
\end{aligned}
$$

which can be written as the autoregressive process

$$
x_{t}^{q}=T x_{t-1}^{q}+R \varepsilon_{t}
$$

with matrices 


$$
T=\left(\begin{array}{ccc}
0 & 0 & \phi \\
0 & 0 & \phi^{2} \\
0 & 0 & \phi^{3}
\end{array}\right), \quad R=\left(\begin{array}{ccc}
1 & 0 & 0 \\
\phi & 1 & 0 \\
\phi^{2} & \phi & 1
\end{array}\right)
$$

where the variance matrix of the vector $x_{t}^{q}$, conditional on $x_{t-1}^{q}$, is equal to $\sigma_{\varepsilon}^{2} R R^{\prime}$. This autoregressive process is equal to the linear Gaussian state space model

$$
\begin{aligned}
x_{t} & =Z \alpha_{t}+\varepsilon_{t}, & & \varepsilon_{t} \sim N(0, H) \\
\alpha_{t+1} & =T \alpha_{t}+R \varepsilon_{t}, & & \varepsilon_{t} \sim N(0, Q)
\end{aligned}
$$

with $Z=I_{3}$, with $\alpha_{t}=x_{t}^{q}$ and $H=0$.

The unconditional variance and covariances (used for initialization of the Kalman Filter) can be obtained from the Yule-Walker equations and are equal to

$$
\begin{aligned}
\gamma_{0} & =\frac{\sigma_{\varepsilon}^{2}}{\left(1-\phi^{2}\right)} \\
\gamma_{1} & =\phi \gamma_{0} \\
\gamma_{2} & =\phi \gamma_{1}
\end{aligned}
$$

\section{A.2 AR(2) PROCESS With STACKED OBSERVATIONS}

For an $\operatorname{AR}(2)$ process with monthly observed variable $x_{\tau}^{m}$ the transformations are similar. Consider the model

$$
x_{\tau}^{m}=\phi_{1} x_{\tau-1}^{m}+\phi_{2} x_{\tau-2}^{m}+\varepsilon_{\tau}, \quad \varepsilon_{\tau} \sim N I D\left(0, \sigma_{\varepsilon}^{2}\right)
$$

The equations of the $\mathrm{AR}(2)$ process for the stacked quarterly observations become

$$
\begin{aligned}
x_{t, 1}^{q} & =\phi_{1} x_{t-1,3}^{q}+\phi_{2} x_{t-1,2}^{q}+\varepsilon_{t, 1} \\
x_{t, 2}^{q} & =\phi_{1} x_{t, 1}^{q}+\phi_{2} x_{t-1,3}^{q}+\varepsilon_{t, 2} \\
x_{t, 3}^{q} & =\phi_{1} x_{t, 2}^{q}+\phi_{2} x_{t, 1}^{q}+\varepsilon_{t, 3}
\end{aligned}
$$

Substitution of the first equation for the value of $x_{t, 1}^{q}$ and the second equation for $x_{t, 2}^{q}$ gives the following set of equations

$$
\begin{aligned}
x_{t, 1}^{q} & =\phi_{1} x_{t-1,3}^{q}+\phi_{2} x_{t-1,2}^{q}+\varepsilon_{t, 1} \\
x_{t, 2}^{q} & =\phi_{1}\left(\phi_{1} x_{t-1,3}^{q}+\phi_{2} x_{t-1,3}^{q}+\varepsilon_{t, 1}\right)+\phi_{2} x_{t-1,2}^{q}+\varepsilon_{t, 2} \\
& =\left(\phi_{1}^{2}+\phi_{2}\right) x_{t-1,3}^{q}+\phi_{1} \phi_{2} x_{t-1,2}^{q}+\phi \varepsilon_{t, 1}+\varepsilon_{t, 2} \\
x_{t, 3}^{q} & =\phi_{1}\left(\left(\phi_{1}^{2}+\phi_{2}\right) x_{t-1,3}^{q}+\phi_{1} \phi_{2} x_{t-1,2}^{q}+\phi \varepsilon_{t, 1}+\varepsilon_{t, 2}\right)+\phi_{2}\left(\phi_{1} x_{t-1,3}^{q}+\phi_{2} x_{t-1,2}^{q}+\varepsilon_{t, 1}\right)+\varepsilon_{t, 3} \\
& =\left(\phi_{1}^{3}+2 \phi_{1} \phi_{2}\right) x_{t-1,3}^{q}+\left(\phi_{1}^{2} \phi_{2}+\phi_{2}^{2}\right) x_{t-1,2}^{q}+\left(\phi_{1}^{2}+\phi_{2}\right) \varepsilon_{t, 1}+\phi_{1} \varepsilon_{t, 2}+\varepsilon_{t, 3}
\end{aligned}
$$

which can be written as the linear Gaussian state space model(35) with matrices 


$$
T=\left(\begin{array}{ccc}
0 & \phi_{2} & \phi_{1} \\
0 & \phi_{1} \phi_{2} & \phi_{1}^{2}+\phi_{2} \\
0 & \phi_{1}^{2} \phi_{2}+\phi_{2}^{2} & \phi_{1}^{3}+2 \phi_{1} \phi_{2}
\end{array}\right), \quad R=\left(\begin{array}{ccc}
1 & 0 & 0 \\
\phi_{1} & 1 & 0 \\
\phi_{1}^{2}+\phi_{2} & \phi_{1} & 1
\end{array}\right)
$$

where the variance matrix of the vector $x_{t}^{q}$, conditional on $x_{t-1}^{q}$, is equal to $\sigma_{\varepsilon}^{2} R R^{\prime}$. The unconditional variance and covariances are equal to

$$
\begin{aligned}
\gamma_{0} & =\frac{1-\phi_{2}}{1+\phi_{2}}\left(\frac{\sigma_{\varepsilon}^{2}}{\left(\phi_{1}+\phi_{2}-1\right)\left(\phi_{2}-\phi_{1}-1\right)}\right) \\
\gamma_{1} & =\frac{\phi_{1}}{\left(1-\phi_{2}\right)} \gamma_{0} \\
\gamma_{2} & =\phi_{1} \gamma_{1}+\phi_{2} \gamma_{0}
\end{aligned}
$$

\section{A.3 AR(3) PROCESS WITH STACKED OBSERVATIONS}

For an $\operatorname{AR}(3)$ process with monthly observed variable $x_{\tau}^{m}$ the transformations are again similar. Consider the model

$$
x_{\tau}^{m}=\phi_{1} x_{\tau-1}^{m}+\phi_{2} x_{\tau-2}^{m}+\phi_{3} x_{\tau-3}^{m}+\varepsilon_{\tau}, \quad \varepsilon_{\tau} \sim N I D\left(0, \sigma_{\varepsilon}^{2}\right)
$$

The equations of the $\operatorname{AR}(3)$ process for the stacked quarterly observations $x_{t}^{q}$ become

$$
\begin{aligned}
x_{t, 1}^{q} & =\phi_{1} x_{t-1,3}^{q}+\phi_{2} x_{t-1,2}^{q}+\phi_{3} x_{t-1,1}^{q}+\varepsilon_{t, 1} \\
x_{t, 2}^{q} & =\phi_{1} x_{t, 1}^{q}+\phi_{2} x_{t-1,3}^{q}+\phi_{3} x_{t-1,2}^{q}++\varepsilon_{t, 2} \\
x_{t, 3}^{q} & =\phi_{1} x_{t, 2}^{q}+\phi_{2} x_{t, 1}^{q}+\phi_{3} x_{t-1,3}^{q}++\varepsilon_{t, 3}
\end{aligned}
$$

Substitutions similar to those described in the previous subsections can again be applied. We have

$$
\begin{aligned}
x_{t, 1}^{q} & =\phi_{1} x_{t-1,3}^{q}+\phi_{2} x_{t-1,2}^{q}+\phi_{3} x_{t-1,1}^{q}+\varepsilon_{t, 1} \\
x_{t, 2}^{q} & =\phi_{1} x_{t, 1}^{q}+\phi_{2} x_{t-1,3}^{q}+\phi_{3} x_{t-1,2}^{q}+\varepsilon_{t, 1} \\
& =\phi_{1}\left(\phi_{1} x_{t-1,3}^{q}+\phi_{2} x_{t-1,2}^{q}+\phi_{3} x_{t-1,1}^{q}+\varepsilon_{t, 1}\right)+\phi_{2} x_{t-1,3}^{q}+\phi_{3} x_{t-1,2}^{q}+\varepsilon_{t, 2} \\
& =\left(\phi_{1}^{2}+\phi_{2}\right) x_{t-1,3}^{q}+\left(\phi_{1} \phi_{2}+\phi_{3}\right) x_{t-1,2}^{q}+\phi_{1} \phi_{3} x_{t-1,1}^{q}+\phi \varepsilon_{t, 1}+\varepsilon_{t, 2} \\
x_{t, 3}^{q} & =\phi_{1} x_{t, 2}^{q}+\phi_{2} x_{t, 1}^{q}+\phi_{3} x_{t-1,3}^{q}+\varepsilon_{t, 1} \\
& =\phi_{1}\left(\phi_{1} x_{t-1,3}^{q}+\phi_{2} x_{t-1,2}^{q}+\phi_{3} x_{t-1,1}^{q}+\varepsilon_{t, 1}\right) \\
& +\phi_{2}\left(\left(\phi_{1}^{2}+\phi_{2}\right) x_{t-1,3}^{q}+\left(\phi_{1} \phi_{2}+\phi_{3}\right) x_{t-1,2}^{q}+\phi_{1} \phi_{3} x_{t-1,1}^{q}+\phi \varepsilon_{t, 1}+\varepsilon_{t, 2}\right) \\
& +\phi_{3} x_{t-1,3}^{q}+\varepsilon_{t, 2} \\
& =\left(\phi_{1}^{3}+2 \phi_{1} \phi_{2}+\phi_{3}\right) x_{t-1,3}^{q}+\left(\phi_{1}^{2} \phi_{2}+\phi_{1} \phi_{3}+\phi_{2}^{2}\right) x_{t-1,2}^{q} \\
& +\left(\phi_{1}^{2} \phi_{3}+\phi_{2} \phi_{3}\right) x_{t-1,1}^{q}+\phi \varepsilon_{t, 1}+\varepsilon_{t, 2}
\end{aligned}
$$

which can be written as the linear Gaussian state space model (35) for the quarterly observed $x_{t}^{q}$ with 


$$
T=\left(\begin{array}{ccc}
\phi_{3} & \phi_{2} & \phi_{1} \\
\phi_{1} \phi_{3} & \phi_{1} \phi_{2}+\phi_{3} & \phi_{1}^{2}+\phi_{2} \\
\phi_{1}^{2} \phi_{3}+\phi_{2} \phi_{3} & \phi_{1}^{2} \phi_{2}+\phi_{1} \phi_{3}+\phi_{2}^{2} & \phi_{1}^{3}+2 \phi_{1} \phi_{2}+\phi_{3}
\end{array}\right)
$$

For the $\operatorname{AR}(3)$ process, $R$ is the same matrix as for the $\operatorname{AR}(2)$ process and the variance matrix of the vector $x_{t}^{q}$, conditional on $x_{t-1}^{q}$, is again equal to $\sigma_{\varepsilon}^{2} R R^{\prime}$. The three values of $x_{t}^{q}$ now depend on all three observation of $x_{t-1}^{q}$ and on the $3 \times 1$ vector of disturbances $\varepsilon_{t}$. The unconditional variance and covariances are equal to

$$
\begin{aligned}
\gamma_{0} & =\frac{\sigma_{\varepsilon}^{2}\left(1-\phi_{2}-\phi_{1} \phi_{3}-\phi_{3}^{2}\right)}{\left(1-\phi_{2}-\phi_{3}-\phi_{1}\right)\left(1+\phi_{2}+\phi_{3} \phi_{1}-\phi_{3}^{2}\right)\left(1+\phi_{3}+\phi_{1}-\phi_{2}\right)} \\
\gamma_{1} & =\frac{\left(\phi_{1}+\phi_{2} \phi_{3}\right) \gamma_{0}}{\left(1-\phi_{2}-\phi_{3} \phi_{1}-\phi_{3}^{2}\right)} \\
\gamma_{2} & =\phi_{1} \gamma_{1}+\phi_{2} \gamma_{0}+\phi_{3} \gamma_{1}
\end{aligned}
$$

\section{A.4 $\operatorname{AR}(p)$ PROCESS With STACKED OBSERVATIONS}

For $\operatorname{AR}(p)$ processes of order $p>3$ the state vector in (35) has to be extended with more lags of $x_{t}^{q}$. For example, for the $\operatorname{AR}(4)$ process we would have $\alpha_{t}=\left(x_{t-1,3}^{q}, x_{t, 1}^{q}, x_{t, 3}^{q}, x_{t, 3}^{q}\right)^{\prime}$ and the transition matrix becomes

$$
T=\left(\begin{array}{cccc}
0 & 0 & 0 & 1 \\
\phi_{4} & \phi_{3} & \phi_{2} & \phi_{1} \\
\phi_{1} \phi_{4} & \phi_{1} \phi_{3}+\phi_{4} & \phi_{1} \phi_{2}+\phi_{3} & \phi_{1}^{2}+\phi_{2} \\
\phi_{1}^{2} \phi_{4}+\phi_{2} \phi_{4} & \phi_{1}^{2} \phi_{3}+\phi_{2} \phi_{3}+\phi_{1} \phi_{4} & \phi_{1}^{2} \phi_{2}+\phi_{1} \phi_{3}+\phi_{2}^{2}+\phi_{4} & \phi_{1}^{3}+2 \phi_{1} \phi_{2}+\phi_{3}
\end{array}\right)
$$

where the variance matrix of the process is defined as $\sigma_{\varepsilon}^{2} R R^{\prime}$ with

$$
R=\left(\begin{array}{cccc}
0 & 0 & 0 & 0 \\
0 & 1 & 0 & 0 \\
0 & \phi_{1} & 1 & 0 \\
0 & \phi_{1}^{2}+\phi_{2} & \phi_{1} & 1
\end{array}\right)
$$

The unconditional variances and covariances can be obtained analytically using the Yule-Walker equations, or by numerically solving the Algebraic Riccati Equation.

\section{B Proofs of Theorems and Propositions}

Proof of Proposition 1. Let $\left\{f_{\tau}\left(f_{1}^{m}\right)\right\}_{\tau \in \mathbb{N}}$ be generated according to (12) with initialization $f_{1}^{m}$ and $\left\|T_{f}\right\|<1,\left\|R_{f}\right\|<\infty$ and $\left\|\Sigma_{\eta}^{2}\right\|<\infty$. Then by Theorem 3.1 in Bougerol (1993), $\left\{f_{\tau}\left(f_{1}^{m}\right)\right\}_{t \in \mathbb{N}}$ converges to an SE sequence $\left\{f_{\tau}\right\}_{t \in \mathbb{Z}}$ satisfying $\mathbb{E}\left|f_{\tau}\right|^{r}<\infty \forall r$. Uniqueness of the limit SE sequence is obtained in Straumann and Mikosch (2006). Furthermore, since $\left\{f_{t}\right\}$ is a linear Gaussian process with $\mathbb{E}\left|x_{\tau}^{m}\right|^{r}<\infty \forall r>0$. The bounds $\left|\beta_{x}\right|<\infty$ and $0<\sigma_{\epsilon}^{2}<\infty$, together with the iid Gaussian nature of the innovations $\left\{\epsilon_{\tau}\right\}^{m}$ 
ensure that $\left\{x_{\tau}^{m}\right\}$ is SE and Gaussian with bounded moments of any order. Similarly, the bounds $\left|\beta_{y}\right|<\infty$ and $0<\sigma_{\zeta}^{2}<\infty$ and the iid Gaussian nature of $\left\{\zeta_{t}\right\}$ ensure the SE linear Gaussian nature of $\left\{y_{t}\right\}$ with $\mathbb{E}\left|y_{t}\right|^{r}<\infty \forall r>0$.

Proof of Theorem 1. For every given $w \in[0,1]$, the random likelihood function $\mathcal{L}_{T}\left(\cdot, \tilde{f}_{1}^{m}\right)$ is trivially almost surely continuous on $\Psi$. The compactness of $\Psi$ implies by Weierstrass' theorem that the arg max set is almost surely non-empty. As a result, $\hat{\psi}_{T}$ exists almost surely $\forall T \in \mathbb{N}$. The continuity of the likelihood function in $f_{\tau}^{m}, x_{\tau}^{m}$ and $y_{t}$ for every $\psi \in \Psi$ implies also measurability of the likelihood under the Borel $\sigma$-algebra. For every given $w \in[0,1]$ the measurability of the WML estimator can now be obtained by application of Theorem 2.11 of White (1994) or Lemma 2.1 and Theorem 2.2 in Gallant and White (1988).

Proof of Theorem 2. The consistency of the WML estimator can be obtained by appealing to the classical extremum estimation theory found e.g. in Theorem 3.4 of White (1994) or Theorem 3.3 of Gallant and White (1988). In particular, for any weight $w \in(0,1]$ and initialization $\tilde{f}_{1}^{m}$, the consistency $\hat{\psi}_{T}\left(w, \tilde{f}_{1}^{m}\right) \stackrel{\text { a.s. }}{\rightarrow} \psi_{0}$ follows from the uniform convergence of the weighted likelihood

$$
\sup _{\psi \in \Psi}\left|\mathcal{L}_{T}\left(\psi, w, \tilde{f}_{1}^{m}\right)-\mathcal{L}_{\infty}(\psi, w)\right| \stackrel{a . s .}{\rightarrow} 0 \forall \tilde{f}_{1}^{m} \in \mathbb{R}_{+} \text {as } T \rightarrow \infty
$$

and the identifiable uniqueness of the true parameter $\psi_{0} \in \Psi$ defined e.g. in White (1994).

To establish the uniform convergence of $\mathcal{L}_{T}\left(\psi, w, \tilde{f}_{1}^{m}\right)$ we use the norm sub-additivity inequality

$$
\sup _{\psi \in \Psi}\left|\mathcal{L}_{T}\left(\psi, w, \tilde{f}_{1}^{m}\right)-\mathcal{L}_{\infty}(\psi, w)\right| \leq \sup _{\psi \in \Psi}\left|\mathcal{L}_{T}\left(\psi, w, \tilde{f}_{1}^{m}\right)-\mathcal{L}_{T}(\psi, w)\right|+\sup _{\psi \in \Psi}\left|\mathcal{L}_{T}(\psi, w)-\mathcal{L}_{\infty}(\psi, w)\right|
$$

where $\mathcal{L}_{T}(\psi, w)$ denotes the likelihood evaluated at the filtered $\tilde{f}_{\tau}(\psi)$ starting in the infinite past. The term

$$
\sup _{\psi \in \Psi}\left|\mathcal{L}_{T}\left(\psi, w, \tilde{f}_{1}^{m}\right)-\mathcal{L}_{T}(\psi, w)\right|
$$

vanishes by the assumption that $\left\|\tilde{f}_{t}^{m}\left(\psi, w, \tilde{f}_{1}^{m}\right)-\tilde{f}_{t}^{m}(\psi)\right\| \stackrel{\text { a.s. }}{\rightarrow} 0$, the continuity of the likelihood function and the continuous mapping theorem.

The ergodic theorem for separable Banach spaces of Rao (1962) ensures that

$$
\sup _{\psi \in \Psi}\left|\mathcal{L}_{T}(\psi, w)-\mathcal{L}_{\infty}(\psi, w)\right| \stackrel{\text { a.s. }}{\rightarrow} 0
$$

(see also Theorem 2.7 in Straumann and Mikosch (2006)) for the sequence $\left\{\mathcal{L}_{T}(\cdot, w)\right\}$ of points in $\mathbb{C}(\Psi, \mathbb{R})$ under:

(i) the SE nature of $\left\{\mathcal{L}_{T}(\cdot, w)\right\}_{T \in \mathbb{Z}}$ which is ensured by SE nature of $\left\{\tilde{f}_{\tau}^{m}\right\}_{\tau \in \mathbb{Z}},\left\{x_{\tau}^{m}\right\}_{\tau \in \mathbb{Z}}$ and $\left\{y_{t}\right\}_{T \in \mathbb{Z}}$, by the continuity of and Proposition 4.3 in Krengel (1985); 
(ii) the moment bound $\mathbb{E} \sup _{\psi \in \Psi}\left|\ell_{t}(\psi, w)\right|<\infty$ ensured by the Gaussian log likelihood under the bounded second moment of $\tilde{f}_{\tau}^{m}, x_{\tau}^{m}$ and $y_{t}$.

The identifiable uniqueness of the true parameter $\psi_{0} \in \Psi$, typically defined as

$$
\sup _{\psi:\left\|\psi-\psi_{0}\right\|>\epsilon} \ell_{\infty}(\psi, w)<\ell_{\infty}\left(\psi_{0}, w\right) \forall \epsilon>0
$$

is ensured by the uniqueness of $\psi_{0}$, the compactness of $\Psi$, and the continuity of $\mathbb{E} \ell_{t}(\psi, w)$ on $\Psi$, which is obtained through the continuity of $\mathcal{L}_{T}$ on $\Psi$ for every $T \in \mathbb{N}$ and the uniform convergence of the likelihood; see e.g. White (1994). The uniqueness of $\psi_{0}$ as the maximizer $\mathcal{L}_{\infty}(\cdot, w)$ for any $w \in(0,1]$ is ensured by Theorem 3 which shows that the maximizer $\mathcal{L}_{\infty}\left(\psi_{0}, w\right)=0$ if and only if $\mathcal{L}_{\infty}\left(\psi_{0}, 1\right)=0$.

Proof of Theorem 3. The consistency statement follows by the same steps as the proof of Theorem 2 with the exception that the SE nature of $\left\{y_{t}\right\}$ is assumed rather than derived through Proposition 1.

Let $z_{t}$ denote a $d_{z}$-variate random vector with joint density $p\left(z_{t}\right)$. Furthermore, consider a family of parametric joint densities indexed by the parameter vector $\psi$, defined as $Q(\Psi):=\left\{q\left(z_{t} ; \psi\right), \psi \in \Psi\right\}$. Note that it is possible but not necessary that $p\left(z_{t}\right) \in Q(\Psi)$. If $\psi_{0}^{*}(1)$ is the pseudo-true parameter that maximizes the limit log likelihood function

$$
\psi_{0}^{*}:=\arg \max _{\psi \in \Psi} \mathbb{E}_{0} \log q\left(z_{t} ; \psi\right)
$$

then it is well known that $\psi_{0}^{*}$ also minimizes the the Kullback-Leibler divergence $\operatorname{KL}(p, q(\cdot ; \psi))$ between $p\left(z_{t}\right)$ and $q\left(z_{t} ; \psi\right)$ because

$$
\arg \max _{\psi \in \Psi} \mathbb{E}_{0} \log q\left(z_{t} ; \psi\right)=\arg \min _{\psi \in \Psi} \mathbb{E}_{0} \log p\left(z_{t}\right)-\mathbb{E}_{0} \log q\left(z_{t} ; \psi\right)=\arg \min _{\psi \in \Psi} \operatorname{KL}(p, q(\cdot ; \psi)) .
$$

Let now the joint density $q\left(x_{t} ; \psi\right)$ be factorized into

$$
q_{1}\left(z_{1, t} \mid z_{2: d_{z}, t} ; \psi_{0}\right) \times q_{2}\left(z_{2: d_{z}, t} ; \psi_{0}\right):=q_{1}\left(z_{1, t} \mid z_{2, t}, \ldots, z_{d_{z}, t} ; \psi_{0}\right) \times q_{2}\left(z_{2, t}, z_{3, t}, \ldots, z_{d_{z}, t} ; \psi_{0}\right)
$$

and define $\psi_{0}^{*}(w)$ as the pseudo-true parameter that maximizes the weighted limit log likelihood function

$$
\psi_{0}^{*}(w):=\arg \max _{\psi \in \Psi} \mathbb{E}_{0} \log q_{1}\left(z_{1, t} \mid z_{2: d_{z}, t} ; \psi\right)+w \log \mathbb{E}_{0} q_{2}\left(z_{2: d_{z}, t} ; \psi\right)
$$

Then it follows naturally that $\psi_{0}^{*}(w)$ is the minimizer of the weighted average of KL divergences

$$
\psi_{0}^{*}(w)=\arg \min _{\psi \in \Psi} \mathrm{KL}\left(q_{1}, p_{1}\right)+w \mathrm{KL}\left(q_{2}, p_{2}\right)
$$


because

$$
\begin{aligned}
\arg \max _{\psi \in \Psi}\left[\mathbb{E}_{0} \log q_{1}\left(z_{1, t} \mid z_{2: d_{z}, t} ; \psi\right)+w \log \mathbb{E}_{0} q_{2}\left(z_{2: d_{z}, t} ; \psi\right)\right] \\
=\arg \max _{\psi \in \Psi}\left[\mathbb{E}_{0} \log q_{1}\left(z_{1, t} \mid z_{2: d_{z}, t} ; \psi\right)-\mathbb{E}_{0} \log p_{1}\left(z_{1, t} \mid z_{2: d_{z}, t}\right)\right. \\
\left.+w \log \mathbb{E}_{0} q_{2}\left(z_{2: d_{z}, t} ; \psi\right)-w \log \mathbb{E}_{0} p_{2}\left(z_{\left.2: d_{z}, t\right)}\right)\right] \\
=\arg \min _{\psi \in \Psi}\left[\mathbb{E}_{0} \log p_{1}\left(z_{1, t} \mid z_{2: d_{z}, t}\right)-\mathbb{E}_{0} \log q_{1}\left(z_{1, t} \mid z_{2: d_{z}, t} ; \psi\right)\right. \\
\left.+w\left(\log \mathbb{E}_{0} p_{2}\left(z_{2: d_{z}, t}\right)-\log \mathbb{E}_{0} q_{2}\left(z_{2: d_{z}, t} ; \psi\right)\right)\right] \\
=\arg \min _{\psi \in \Psi}\left[\operatorname{KL}\left(q_{1}(\cdot ; \psi), p_{1}\right)+w \operatorname{KL}\left(q_{2}(\cdot ; \psi), p_{2}\right)\right] .
\end{aligned}
$$

Clearly, if $w=1$, then we obtain the usual ML pseudo-true parameter since

$$
\psi_{0}^{*}(1)=\arg \min _{\psi \in \Psi} \operatorname{KL}\left(q_{1}(\cdot ; \psi), p_{1}\right)+\mathrm{KL}\left(q_{2}(\cdot ; \psi), p_{2}\right)=\arg \min _{\psi \in \Psi} \mathrm{KL}(q(\cdot ; \psi), p) .
$$

For $w=0$ we obtain the ML estimator for the conditional model

$$
\psi_{0}^{*}(0)=\arg \min _{\psi \in \Psi} \operatorname{KL}\left(q_{1}(\cdot ; \psi), p_{1}\right)
$$

Finally, it is also clear that the transformed $\operatorname{KL}$ divergence $\operatorname{TKL}(q, p):=\operatorname{KL}\left(q_{1}(\cdot ; \psi), p_{1}\right)+$ $w \mathrm{KL}\left(q_{2}(\cdot ; \psi), p_{2}\right)$ satisfies

$$
\mathrm{KL}\left(q_{1}(\cdot ; \psi), p(\cdot ; \psi)\right) \leq \operatorname{TKL}(q(\cdot ; \psi), p) \leq \operatorname{KL}(q(\cdot ; \psi), p)
$$

and that $\operatorname{TKL}(q(\cdot ; \psi), p)$ is a pre-metric for any $w \in(0,1]$ as it inherits positivity $\operatorname{TKL}(q(\cdot ; \psi), p) \geq$ 0 from the positivity of the KL divergence, and satisfies also the identity of indiscernibles since

$$
\operatorname{TKL}(q(\cdot ; \psi), p)=\operatorname{KL}\left(q_{1}(\cdot ; \psi), p_{1}\right)+w \operatorname{KL}\left(q_{2}(\cdot ; \psi), p_{2}\right)=0
$$

if and only if $q_{1}(\cdot ; \psi)=p_{1}$ and $q_{2}(\cdot ; \psi)=p_{2}$, and hence, if and only if $q(\cdot ; \psi)=p$.

Proof of Theorem 4. Asymptotic normality of the WML estimator can be obtained by verifying the conditions of Theorem 6.2 of White (1994):

(i) $\hat{\psi}_{T}\left(\tilde{f}_{1}^{m}, w\right) \stackrel{\text { a.s. }}{\rightarrow} \psi_{0} \in \operatorname{int}(\Psi)$;

(ii) $\mathcal{L}_{T}\left(\cdot, w, \tilde{f}_{1}^{m}\right) \in \mathbb{C}^{2}(\Psi)$ a.s.;

(iii) $\sqrt{T} \mathcal{L}_{T}^{\prime}\left(\psi_{0}, w, \tilde{f}_{1}^{m}, \tilde{d f}_{1}^{m}\right) \stackrel{d}{\rightarrow} N\left(0, \mathcal{J}\left(\psi_{0}, w\right)\right)$;

(iv) $\sup _{\psi \in \Psi}\left\|\mathcal{L}_{T}^{\prime \prime}\left(\psi, w, \tilde{f}_{1}^{m}, \tilde{d} f_{1}^{m}, \tilde{d d} f_{1}^{m}\right)-\ell_{\infty}^{\prime \prime}(\psi, w)\right\| \stackrel{a . s .}{\rightarrow} 0 ;$

(v) $\ell_{\infty}^{\prime \prime}(\psi, w)=\mathbb{E} \ell_{t}^{\prime \prime}(\psi, w)=\mathcal{I}(\psi, w)$ is non-singular 
(i) and (ii) follow naturally from Theorem 2, the additional assumption that $\psi_{0} \in$ $\operatorname{int}(\Psi)$ and the differentiability of the Gaussian likelihood.

(iii) follows by the asymptotic SE nature of the score $\left\{\ell_{t}^{\prime}\left(\psi, w, \tilde{f}_{1}^{m}, \tilde{d} f_{1}^{m}\right)\right\}$ which is implied by the SE nature of $\left\{x_{\tau}^{m}\right\}$ and $\left\{y_{t}\right\}$, and the asymptotic SE nature of the filtered sequence $\left\{\tilde{f}_{\tau}\left(\psi, \tilde{f}_{1}^{m}\right)\right\}$ and its derivative $\left\{\tilde{d} f_{\tau}\left(\psi, \tilde{d} f_{1}^{m}\right)\right\}$. Since the limit score sequence $\left\{\ell_{t}^{\prime}(\psi, w)\right\}$ is SE we can apply the CLT for SE martingales in Billingsley (1961) to obtain

$$
\sqrt{T} \mathcal{L}_{T}^{\prime}\left(\psi_{0}, w\right) \stackrel{d}{\rightarrow} N\left(0, \mathcal{J}\left(\psi_{0}, w\right)\right) \text { as } T \rightarrow \infty
$$

where $\mathcal{J}\left(\psi_{0}, w\right)=\mathbb{E}\left(\ell_{t}^{\prime}\left(\psi_{0}, w\right) \ell_{t}^{\prime}\left(\psi_{0}, w\right)^{\top}\right)<\infty$. By Theorem 18.10[iv] in van der Vaart (2000) it thus follows that

$$
\sqrt{T} \mathcal{L}_{T}^{\prime}\left(\psi_{0}, w, \tilde{f}_{1}^{m}, \tilde{d f}_{1}^{m}\right) \stackrel{d}{\rightarrow} N\left(0, \mathcal{J}\left(\psi_{0}, w\right)\right) \text { as } T \rightarrow \infty
$$

as long as

$$
\left\|\mathcal{L}_{T}^{\prime}\left(\psi_{0}, w, \tilde{f}_{1}^{m}, \tilde{d f}{ }_{1}^{m}\right)-\mathcal{L}_{T}^{\prime}\left(\psi_{0}, w\right)\right\| \stackrel{\text { e.a.s. }}{\rightarrow} 0 \text { as } T \rightarrow \infty
$$

since (51) ensures $\sqrt{T}\left\|\mathcal{L}_{T}^{\prime}\left(\psi_{0}, w, \tilde{f}_{1}^{m}, \tilde{d f}_{1}^{m}\right)-\mathcal{L}_{T}^{\prime}\left(\psi_{0}, w\right)\right\| \stackrel{\text { a.s. }}{\rightarrow} 0$ as $T \rightarrow \infty$. The e.a.s. convergence in (51) follows from

$$
\left|f_{t}^{m}\left(\psi_{0}, w, \tilde{f}_{1}^{m}\right)-f_{t}^{m}\left(\psi_{0}, w\right)\right| \stackrel{\text { e.a.s. }}{\rightarrow} 0
$$

and

$$
\left\|\tilde{d} f_{\tau}^{m}\left(\psi_{0}, \tilde{d f} f_{1}^{m}\right)-\tilde{d f} \tau_{\tau}^{m}\left(\psi_{0}\right)\right\| \stackrel{e . a_{-} s .}{\rightarrow} 0 .
$$

Furthermore, since the score of the weighted likelihood is differentiable, we can use the mean-value theorem to obtain

$$
\left\|\mathcal{L}_{T}^{\prime}\left(\psi_{0}, w, \tilde{f}_{1}^{m}, \tilde{d} f_{1}^{m}\right)-\mathcal{L}_{T}^{\prime}\left(\psi_{0}, w\right)\right\| \leq \sum_{j}\left|d \mathcal{L}_{T}^{\prime} \| \tilde{d f}_{j, \tau}^{m}\left(\psi_{0}, \tilde{d f}_{1}^{m}\right)-\tilde{d f}_{j, \tau}^{m}\left(\psi_{0}\right)\right|
$$

where $\tilde{d f}_{j, \tau}^{m}\left(\psi_{0}, \tilde{d f} f_{1}^{m}\right)$ denotes the $j$-th element of $\tilde{d f}_{\tau}^{m}\left(\psi_{0}, \tilde{d f}_{1}^{m}\right)$, and $d \mathcal{L}_{T}^{\prime}$ denotes the derivative $\partial \mathcal{L}_{T}^{\prime}\left(\psi_{0}, w, \tilde{f}_{1}^{m}, \tilde{d f}_{1}^{m}\right) / \partial \tilde{d} f_{j}$ evaluated at some appropriate point between $\tilde{d f}_{j, \tau}^{m}\left(\psi_{0}, \tilde{d} f_{1}^{m}\right)$ and $\tilde{d} f_{j, \tau}^{m}\left(\psi_{0}\right)$. The bounded moments of the weighted likelihood derivatives and the e.a.s. convergence of the filtered process and its derivatives yield

$$
\left\|\mathcal{L}_{T}^{\prime}\left(\psi_{0}, w, \tilde{f}_{1}^{m}, \tilde{d f} f_{1}^{m}\right)-\mathcal{L}_{T}^{\prime}\left(\psi_{0}, w\right)\right\|=\sum_{j} O_{p}(1) o_{e . a . s}(1)=o_{\text {e.a.s. }}(1) .
$$

(iii) follows by noting that

$$
\begin{array}{r}
\left.\sup _{\psi \in \Psi} \| \mathcal{L}_{T}^{\prime \prime}\left(\psi, w, \tilde{f}_{1}^{m}, \tilde{d f}\right]_{1}^{m}, \tilde{d d} f_{1}^{m}\right)-\mathcal{L}_{\infty}^{\prime \prime}(\psi, w) \leq \sup _{\psi \in \Psi}\left\|\mathcal{L}_{T}^{\prime \prime}\left(\psi, w, \tilde{f}_{1}^{m}, \tilde{d} f_{1}^{m}, d \tilde{d} f_{1}^{m}\right)-\mathcal{L}_{T}^{\prime \prime}(\psi, w)\right\| \\
+\sup _{\psi \in \Psi}\left\|\mathcal{L}_{T}^{\prime \prime}(\psi, w)-\mathcal{L}_{\infty}^{\prime \prime}(\psi, w)\right\| .
\end{array}
$$


Clearly, $\sup _{\psi \in \Psi}\left\|\mathcal{L}_{T}^{\prime \prime}\left(\psi, w, \tilde{f}_{1}^{m}, \tilde{d} f_{1}^{m}, \tilde{d d} f_{1}^{m}\right)-\mathcal{L}_{T}^{\prime \prime}(\psi, w)\right\| 0$ as $t \rightarrow \infty$ by the continuous mapping theorem and the e.a.s. convergence of the filtered process and its derivatives, and $\sup _{\psi \in \Psi}\left\|\mathcal{L}_{T}^{\prime \prime}(\psi, w)-\mathcal{L}_{\infty}^{\prime \prime}(\psi, w)\right\|$ vanishes by a ULLN under the uniform moment bound on the weighted likelihood $\mathbb{E} \sup _{\psi \in \Psi}\left\|\mathcal{L}_{t}^{\prime \prime}(\psi, w)\right\|<\infty$.

(v) the uniqueness of $\psi_{0}$ as a maximum of $\ell_{\infty}^{\prime \prime}(\psi, w)$ ensures the non-singularity of the limit weighted likelihood $\mathcal{L}_{\infty}^{\prime \prime}(\psi, w)=\mathbb{E} \mathcal{L}_{t}^{\prime \prime}(\psi, w)=\mathcal{I}(\psi, w)$.

Proof of Theorem 5. Follows the same steps as the proof of Theorem 4 ith the exception that the required properties of $\left\{x_{\tau}^{m}\right\}$ and $\left\{y_{t}\right\}$ are directly assumed rather than derived through Proposition 1.

\section{Technichl Appendix: Recursive Algorithms}

In general, observations from the monthly $\operatorname{AR}(p)$ processes of order $p$ can be stacked into quarterly or yearly vectors. The system matrices $T$ and $R$ can both be obtained by recursive algorithms. We will first show how the recursions work for the $\operatorname{AR}(p)$ processes with $p=1,2,3$ that were described in the previous subsection. Then we will give the general steps of the recursion for $\operatorname{AR}(p)$ processes.

$\operatorname{AR}(1)$

We will start with the example of the $\mathrm{AR}(1)$ process in (5) and (6). The transition matrix $T=\left[T_{1}^{\prime}, \ldots, T_{s}^{\prime}\right]^{\prime}$, where $T_{i}$ is the $i$-th row of matrix $T$ for the low frequency autoregressive process in the state space representation, can be constructed as follows. From the equation $x_{t, 1}^{q}=\phi x_{t-1,3}^{q}+\varepsilon_{t, 1}$ we have $T_{1}=[0,0, \phi]$. For the subsequent rows we use the algorithm. Hence, we have $T_{2}=\phi T_{1}=\left[0,0, \phi^{2}\right]$ and $T_{3}=\phi T_{2}=\left[0,0, \phi^{3}\right]$. This may seem very trivial for the $\operatorname{AR}(1)$ process, but similar algorithms can be used for higher order $\operatorname{AR}(p)$ processes as well, as we will show in the next examples.

The matrix $R$ in (6) can be constructed as follows. Start with ones on the diagonal and zeros for all upper-diagonal elements. We refer to the diagonal elements as $R_{D}$. The elements that are one position under the diagonal are denoted as $R_{D+1}$ and are equal to $\phi R_{D}=\phi$. For the elements (in this case only one) two positions under the diagonal we have $R_{D+2}=\phi R_{D+1}=\phi^{2}$.

$\operatorname{AR}(2)$

For the $\operatorname{AR}(2)$ process the matrix $T$ in (7), with $\phi_{3}=0$, can also be constructed with the recursive algorithm. From the equation $x_{t, 1}^{q}=\phi_{1} x_{t-1,3}^{q}+\phi_{2} x_{t-1,2}^{q}+\varepsilon_{t, 1}$ we have $T_{1}=\left[0, \phi_{2}, \phi_{1}\right]$. To construct the second row $T_{2}$ we start with the equation $x_{t, 2}^{q}=$ $\phi_{1} x_{t, 1}^{q}+\phi_{2} x_{t-1,3}^{q}+\varepsilon_{t, 2}$ and substitute the first equation for the value of $x_{t, 1}^{q}$. This gives us 


$$
\begin{aligned}
x_{t, 2}^{q} & =\phi_{1}\left(\phi_{1} x_{t-1,3}^{q}+\phi_{2} x_{t-1,2}^{q}+\varepsilon_{t, 1}\right)+\phi_{2} x_{t-1,2}^{q}+\varepsilon_{t, 2} \\
& =\left(\phi_{1}^{2}+\phi_{2}\right) x_{t-1,3}^{q}+\phi_{1} \phi_{2} x_{t-1,2}^{q}+\phi \varepsilon_{t, 1}+\varepsilon_{t, 2}
\end{aligned},
$$

which gives us $T_{2}=\left[0,\left(\phi_{1} \phi_{2}\right),\left(\phi_{1}^{2}+\phi_{2}\right)\right]$. The third row $T_{3}$ can now be constructed using the algorithm $T_{j}=\phi_{1} T_{j-1}+\phi_{2} T_{j-2}$. In this example, the recursive formula only has to be used for $j=3$.

The matrix $R$ can be constructed as follows. Start with ones on the diagonal and zeros for all upper-diagonal elements. We again refer to the diagonal elements as $R_{D}$. For the elements under the diagonal we have $R_{D+j}=\phi_{1} R_{D+j-1}+\phi_{2} R_{D+j-2}$.

$\operatorname{AR}(3)$

For the $\operatorname{AR}(3)$ process, matrix $R$ is the same matrix as for the $\operatorname{AR}(2)$ process, which can be explained as follows. The elements under the unit diagonal of matrix $R$ are given by the equation $R_{D+j}=\phi_{1} R_{D+j-1}+\phi_{2} R_{D+j-2}+\phi_{3} R_{D+j-3}$. However, because in this example $R$ is only a $3 \times 3$ matrix, the term $\phi_{3} R_{D+j-3}$ is never effectively used.

In this example the elements of all the three rows of $T$ in (7) have to be derived manually. From the equation $x_{t, 1}^{q}=\phi_{1} x_{t-1,3}^{q}+\phi_{2} x_{t-1,2}^{q}+\phi_{3} x_{t-1,1}^{q}+\varepsilon_{t, 1}$ we have $T_{1}=$ $\left[\phi_{3}, \phi_{2}, \phi_{1}\right]$. Substitutions as in (55) are needed to construct $T_{2}$ and $T_{3}$. We have

$$
\begin{aligned}
x_{t, 2}^{q} & =\phi_{1} x_{t, 1}^{q}+\phi_{2} x_{t-1,3}^{q}+\phi_{3} x_{t-1,2}^{q}+\varepsilon_{t, 1} \\
& =\phi_{1}\left(\phi_{1} x_{t-1,3}^{q}+\phi_{2} x_{t-1,2}^{q}+\phi_{3} x_{t-1,1}^{q}+\varepsilon_{t, 1}\right)+\phi_{2} x_{t-1,3}^{q}+\phi_{3} x_{t-1,2}^{q}+\varepsilon_{t, 2} \\
& =\left(\phi_{1}^{2}+\phi_{2}\right) x_{t-1,3}^{q}+\left(\phi_{1} \phi_{2}+\phi_{3}\right) x_{t-1,2}^{q}+\phi_{1} \phi_{3} x_{t-1,1}^{q}+\phi \varepsilon_{t, 1}+\varepsilon_{t, 2}
\end{aligned}
$$

which gives us $T_{2}=\left[\phi_{1} \phi_{3},\left(\phi_{1} \phi_{2}+\phi_{3}\right),\left(\phi_{1}^{2}+\phi_{2}\right)\right]$. The third row $T_{3}$ is constructed as follows

$$
\begin{aligned}
x_{t, 3}^{q} & =\phi_{1} x_{t, 2}^{q}+\phi_{2} x_{t, 1}^{q}+\phi_{3} x_{t-1,3}^{q}+\varepsilon_{t, 1} \\
& =\phi_{1}\left(\phi_{1} x_{t-1,3}^{q}+\phi_{2} x_{t-1,2}^{q}+\phi_{3} x_{t-1,1}^{q}+\varepsilon_{t, 1}\right) \\
& +\phi_{2}\left(\left(\phi_{1}^{2}+\phi_{2}\right) x_{t-1,3}^{q}+\left(\phi_{1} \phi_{2}+\phi_{3}\right) x_{t-1,2}^{q}+\phi_{1} \phi_{3} x_{t-1,1}^{q}+\phi \varepsilon_{t, 1}+\varepsilon_{t, 2}\right) \\
& +\phi_{3} x_{t-1,3}^{q}+\varepsilon_{t, 2} \\
& =\left(\phi_{1}^{3}+2 \phi_{1} \phi_{2}+\phi_{3}\right) x_{t-1,3}^{q}+\left(\phi_{1}^{2} \phi_{2}+\phi_{1} \phi_{3}+\phi_{2}^{2}\right) x_{t-1,2}^{q} \\
& +\left(\phi_{1}^{2} \phi_{3}+\phi_{2} \phi_{3}\right) x_{t-1,1}^{q}+\phi \varepsilon_{t, 1}+\varepsilon_{t, 2}
\end{aligned}
$$

which gives us $T_{3}=\left[\left(\phi_{1}^{2} \phi_{3}+\phi_{2} \phi_{3}\right),\left(\phi_{1}^{2} \phi_{2}+\phi_{1} \phi_{3}+\phi_{2}^{2}\right),\left(\phi_{1}^{3}+2 \phi_{1} \phi_{2}+\phi_{3}\right)\right]$.

The elements of subsequent rows would be given by the equation $T_{j}=\phi_{1} T_{j-1}+$ $\phi_{2} T_{j-2}+\phi_{3} T_{j-3}$. However, we do not need the recursion in this example, since the matrix $T$ only has 3 rows.

\section{AR(3) WITH YEARLY FREQUENCY}

This becomes different when the monthly observations of $x_{\tau}^{m}$ are stacked into a $12 \times 1$ vector with yearly observations $x_{t}^{y}$. The equations for the $\operatorname{AR}(3)$ process then become 


$$
\begin{aligned}
x_{t, 1}^{y} & =\phi_{1} x_{t-1,12}^{y}+\phi_{2} x_{t-1,11}^{y}+\phi_{3} x_{t-1,10}^{y}+\varepsilon_{t, 1}^{y} \\
x_{t, 2}^{y} & =\phi_{1} x_{t, 1}^{y}+\phi_{2} x_{t-1,12}^{y}+\phi_{3} x_{t-1,11}^{y}+\varepsilon_{t, 2}^{y} \\
& \vdots \\
x_{t, 12}^{y} & =\phi_{1} x_{t, 11}^{y}+\phi_{2} x_{t, 10}^{y}+\phi_{3} x_{t, 9}^{y}+\varepsilon_{t, 12}^{y}
\end{aligned}
$$

The $12 \times 12$ matrix $T$ can again be constructed using the equation $T_{j}=\phi_{1} T_{j-1}+$ $\phi_{2} T_{j-2}+\phi_{3} T_{j-3}$, for $j=4, \ldots, 12$ and with

$$
\left(\begin{array}{c}
T_{1} \\
T_{2} \\
T_{3}
\end{array}\right)=\left(\begin{array}{cccccc}
0 & \ldots & 0 & \phi_{3} & \phi_{2} & \phi_{1} \\
0 & \ldots & 0 & \phi_{1} \phi_{3} & \phi_{1} \phi_{2}+\phi_{3} & \phi_{1}^{2}+\phi_{2} \\
0 & \ldots & 0 & \phi_{1}^{2} \phi_{3}+\phi_{2} \phi_{3} & \phi_{1}^{2} \phi_{2}+\phi_{1} \phi_{3}+\phi_{2}^{2} & \phi_{1}^{3}+2 \phi_{1} \phi_{2}+\phi_{3}
\end{array}\right)
$$

where the last three columns are equal to the matrix $T$ of the quarterly $\operatorname{AR}(3)$ process in (7).

The matrix $R$ is again constructed using $R_{D+j}=\phi_{1} R_{D+j-1}+\phi_{2} R_{D+j-2}+\phi_{3} R_{D+j-3}$ for $j=1, \ldots, 11$ and starting with the $12 \times 12$ identity matrix $I_{12}$.

$\operatorname{AR}(p)$

To summarize, for monthly $\operatorname{AR}(p)$ processes of order $p$ that are stacked into quarterly or yearly vectors the transition matrix $T$ of the autoregressive process and state space representation is obtained by the following recursive algorithm:

- Construct the first $p$ rows of $T$ by rewriting the equations for the first $p$ elements of $x_{t}^{q}$ in terms of elements of $x_{t-1}^{q}$.

- When the vector length $s$ is larger than $p$, calculate subsequent rows using $T_{j}=$ $\phi_{1} T_{j-1}+\ldots+\phi_{p} T_{j-p}$ for $j=p+1, \ldots, s$ (with $s=3$ for quarterly and $s=12$ for yearly vectors).

The matrix $R$ is obtained by the following recursive algorithm:

- Start with the identity matrix $I_{3}$ (quarterly) or $I_{12}$ (yearly) and refer to the diagonal elements as $R_{D}$.

- Elements $j$ positions under the diagonal are referred to as $R_{D+j}$. Calculate these elements as $R_{D+j}=\phi_{1} R_{D+j-1}+\ldots+\phi_{p} R_{D+j-p}$ for $j=1,2$ (quarterly) or $j=$ $1, \ldots, 11$ (yearly).

Note that when the order $p$ of the $\operatorname{AR}(p)$ process exceeds the vector length $s$, earlier lags have to be added to the state vector. See Section 2.3 for an example with an $\operatorname{AR}(4)$ process and $3 \times 1$ vectors of observations. 


\section{Technical Appendix: Benchmark Models}

\section{D.1 BRIDGE Models}

Bridge Models are linear dynamic equations where the aggregate GDP is explained by suitable short-term indicators. For this comparison we use the same four monthly indicators (log-transformed and demeaned) as in the dynamic factor models described in the previous subsection. The monthly indicators are averaged in quarterly series, such that they have the same frequency as GDP and a linear regression model with GDP as the dependent variable is estimated. For this comparison, we also include two lags of GDP in the equation

$$
y_{t}=\beta_{y, 1} y_{t-1}+\beta_{y, 2} y_{t-2}+\beta_{1} x_{t}^{(1)}+\beta_{2} x_{t}^{(2)}+\beta_{3} x_{t}^{(3)}+\beta_{4} x_{t}^{(4)}+\varepsilon_{t}
$$

where all variables are log-transformed and demeaned, so no constant is needed in the equation.

Forecasting with BM is done in two steps: first the monthly indicators are forecasted up to the desired forecasting horizon, using separate $\operatorname{AR}(2)$ models for the four different indicators. For example, when forecasting the next quarter GDP value $y_{(t+1)}$ and the conditioning indicators are already known for the first month of the quarter $(t+1)$, the indicators have to be forecast two months ahead by the $\operatorname{AR}(2)$ models. In the second step, the forecasts and known values are aggregated to quarterly averages again and plugged into the estimated regression equation, together with the lagged values of GDP. All parameters of the model can be estimated with Least Squares.

\section{D.2 MIDAS REgRESSIONS}

The MIDAS approach was proposed by Ghysels, Santa-Clara, and Valkanov (2006). Similar to in BM, the series with the higher frequency are regressed on the series with the lower frequency. However, when forecasting with MIDAS regression only one step is required. To obtain forecasts at horizon $h$, the values of $y_{t}$ are simply regressed on the values of the indicators up to period $t-h$ and the dynamics of the regressors are not specified by the model.

In MIDAS regressions, the higher frequency series are not aggregated, but each lag has its own regression coefficient. This means that in our example, three regression coefficients have to be estimated per indicator. To avoid parameter proliferation, the coefficients of the different lags are described by a weighting function $w_{j}$, such that the regression equation for this example becomes

$$
y_{t+h}=\beta_{y} \sum_{j=1}^{2} w_{j}\left(\theta_{y}\right) y_{t-j}+\sum_{i=1}^{4} \beta_{x^{(i)}} \sum_{j=1}^{6} w_{j}\left(\theta_{x^{(i)}}\right) x_{t-j / m}^{(i)}+\varepsilon_{t+h}
$$

where $m=3$, because each quarter consists of three months. Again all variables are logtransformed and demeaned, so no constant is needed in the equation. Two lags of $y$ are 
included in the equation, and six lags (e.g. two quarters) for each $x_{i}$. These numbers were chosen in order to make all models under investigation in this comparison use roughly the same amount of information in forecasting. The weighting schemes $w_{j}\left(\theta_{y}\right)$ and $w_{j}\left(\theta_{x_{i}}\right)$ are two-parameter exponential Almon lag polynomials:

$$
w_{j}\left(\theta_{1}, \theta_{2}\right)=\frac{\exp \left(\theta_{1} j+\theta_{2} j^{2}\right)}{\sum_{j=1}^{6} \exp \left(\theta_{1} j+\theta_{2} j^{2}\right)}
$$

Note that the weights are governed by two parameters and scaled such that they add up to one. The parameters can be estimated with Least Squares. When the model is correctly specified and the parameters are known, the Kalman filter is superior to MIDAS by construction. Otherwise, it is under investigation whether MIDAS or the state space method is superior; see the study of Bai, Ghysels, and Wright (2011) where both MIDAS and state space methods are considered. They show under which conditions the methods are identical and provide evidence that the Kalman filter is slightly more accurate.

E Technical Appendix: Weighted Maximum Likelihood: MSE FigURES 
Figure 3: Scenario 1: Underspecification
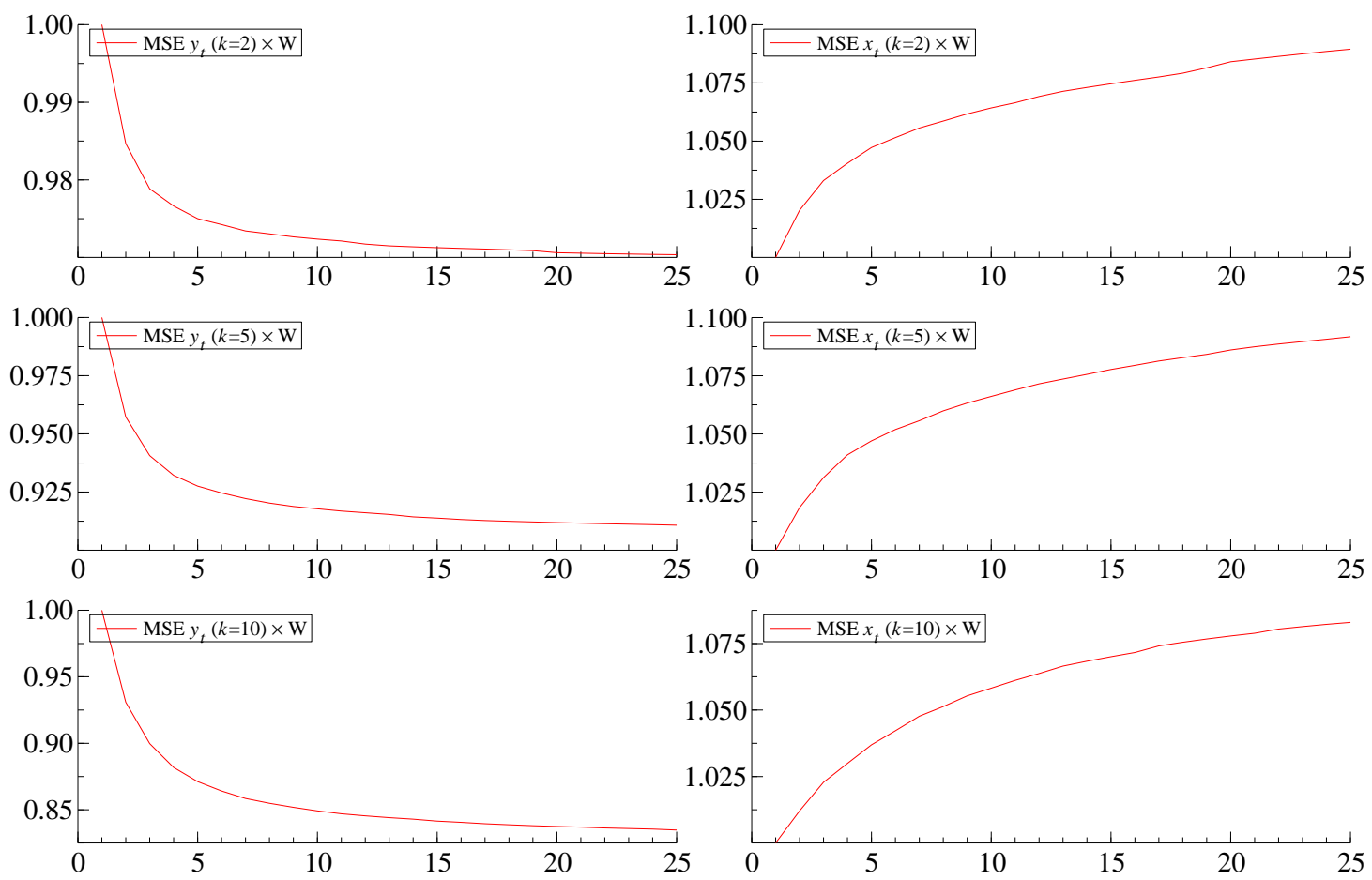

This figure presents the in-sample MSE of the one-step ahead predictions using an underspecified model for different values of $W$ in the weighted loglikelihood function (26) for $k=2$ (upper panels), $k=5$ (middle panels) and $k=10$ (lower panels) variables. The DGP is a common factor model with idiosyncratic factors and the estimated model is a model with only a common factor. The numbers on the horizontal axis represent the value of $W$ and all numbers are relative to the values at $W=1$. The MSEs of the target variable $y_{t}$ are presented in the figures on the right. The panels on the left present the average MSEs of the other variables $x_{t}$. 
Figure 4: Scenario 2: Misspecification
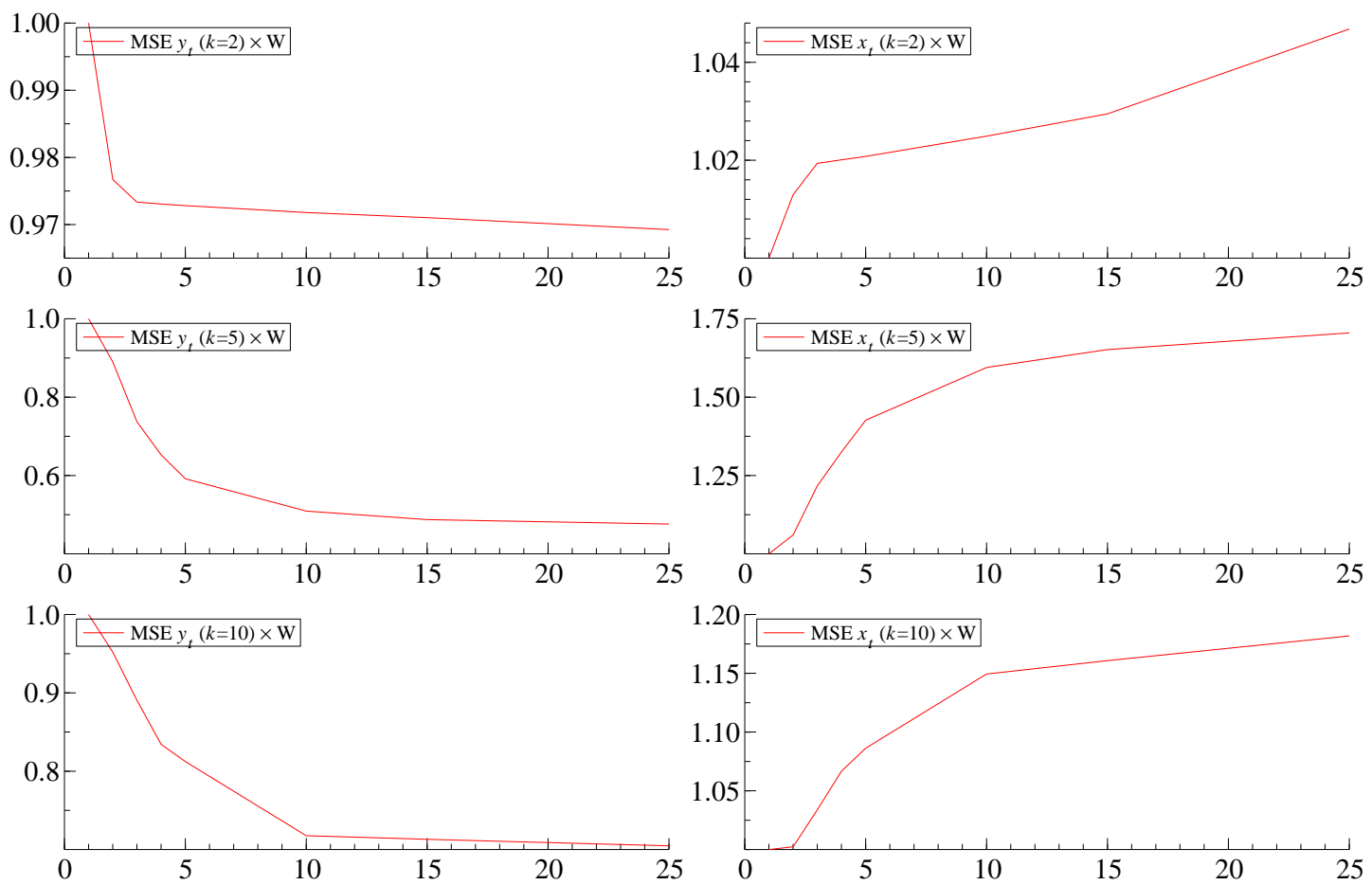

This figure presents the in-sample MSE of the one-step ahead predictions using a misspecified model for different values of $W$ in the weighted loglikelihood function (26) for $k=2$ (upper panels), $k=5$ (middle panels) and $k=10$ (lower panels) variables. The DGP is a VAR(1) model and the estimated model is a dynamic factor model with one common factor. The numbers on the horizontal axis represent the value of $W$ and all numbers are relative to the values at $W=1$. The MSEs of the target variable $y_{t}$ are presented in the figures on the right. The panels on the left present the average MSEs of the other variables $x_{t}$. 
Figure 5: Scenario 3: Correct specification
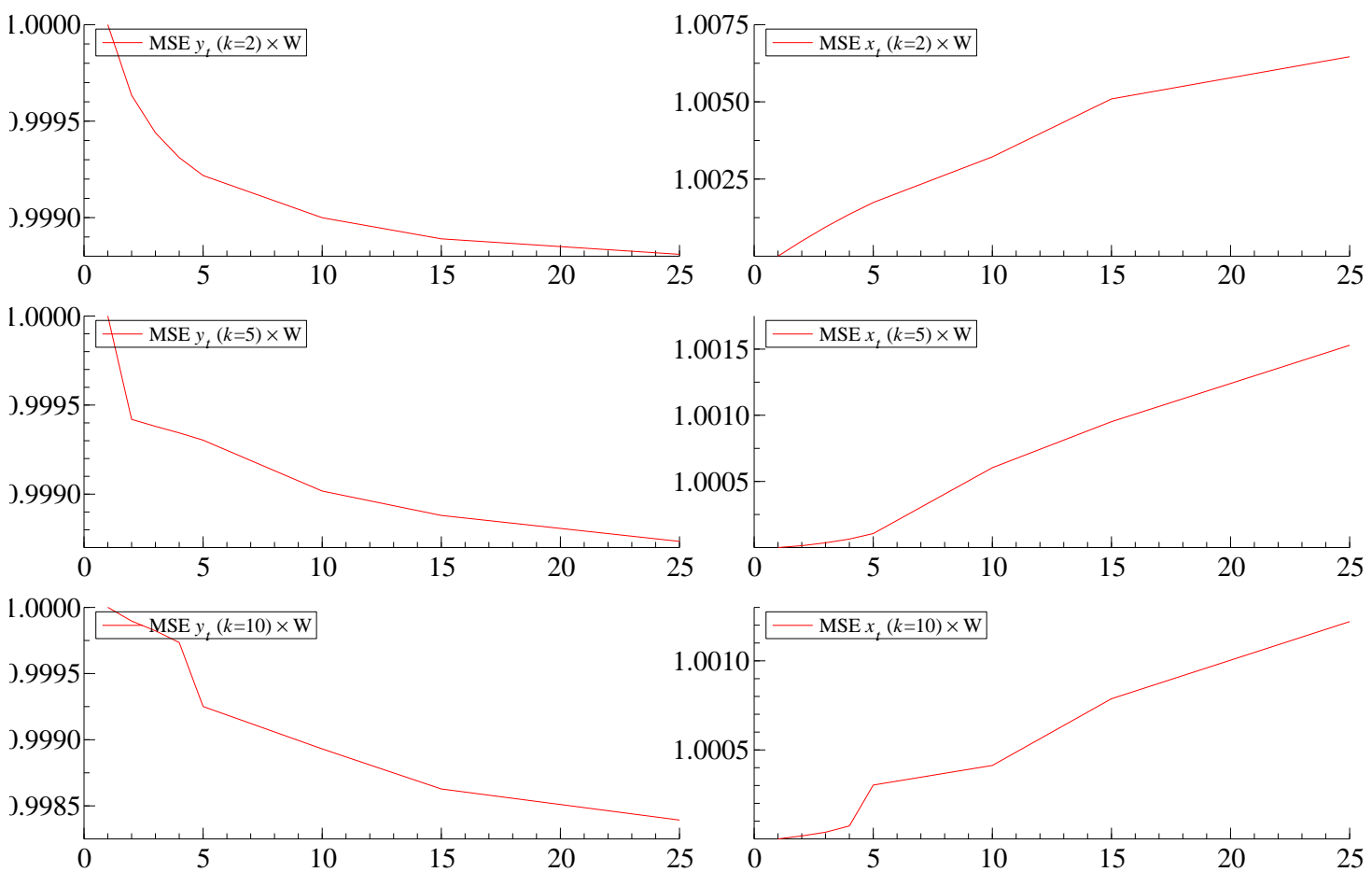

This figure presents the in-sample MSE of the one-step ahead predictions using a correctly specified model for different values of $W$ in the weighted loglikelihood function (26) for $k=2$ (upper panels), $k=5$ (middle panels) and $k=10$ (lower panels) variables. The DGP and the estimated model are both a common factor model with idiosyncratic factors. The numbers on the horizontal axis represent the value of $W$ and all numbers are relative to the values at $W=1$. The MSEs of the target variable $y_{t}$ are presented in the figures on the right. The panels on the left present the average MSEs of the other variables $x_{t}$. 


\section{REFERENCES}

Aruoba, S., F. X. Diebold, and C. Scotti (2008). Real-time measurement of business conditions, second version. PIER Working Paper Archive No. 11, Penn Institute for Economic Research, Department of Economics, University of Pennsylvania. Working paper.

Baffigi, A., R. Golinelli, and G. Parigi (2004). Bridge models to forecast the euro area gdp. International Journal of Forecasting 20, 447-460.

Bai, J., E. Ghysels, and J. H. Wright (2011). State space models and midas regressions. Working paper.

Banbura, M., D. Giannone, M. Modugno, and L. Reichlin (2013). Nowcasting and the real time data flow. Handbook of Economic Forecasting $2 A$.

Billingsley, P. (1961). The lindeberg-levy theorem for martingales. Proceedings of the American Mathematical Society 12(5), 788-792.

Blasques, F., S. J. Koopman, and A. Lucas (2014). Maximum likelihood estimation for generalized autoregressive score models. Technical Report 14-029/III, Tinbergen Institute Discussion Paper.

Bougerol, P. (1993). Kalman filtering with random coefficients and contractions. SIAM Journal on Control and Optimization 31 (4), 942-959.

Diebold, F. X. and R. S. Mariano (1995). Comparing predictive accuracy. JBES 13(3), 253-63.

Durbin, J. and S. J. Koopman (2012). Time Series Analysis by State Space Methods. Oxford: Oxford University Press.

Foroni, C., M. Marcellino, and C. Schumacher (2012). U-midas: Midas regressions with unrestricted lag polynomials. Journal of the Royal Statistical Society. Forthcoming.

Gallant, R. and H. White (1988). A Unified Theory of Estimation and Inference for Nonlinear Dynamic Models. Cambridge University Press.

Ghysels, E., P. Santa-Clara, and R. Valkanov (2006). Predicting volatility: getting the most out of return data sampled at different frequencies. Journal of Econometrics 131, 59-95.

Golinelli, R. G. and Parigi (2007). The use of monthly indicators to forecast quarterly gdp in the short-run: An application to the g7 countries. Journal of Forecasting 26, $77-94$.

Koopman, S. J. and J. Durbin (2000). Fast filtering and smoothing for multivariate state space models. Journal of Time Series Analysis 21, 281-96.

Krengel, U. (1985). Ergodic theorems. Berlin: De Gruyter studies in Mathematics.

Marcellino, M., A. Carriero, and T. E. Clark (2014). Real-time nowcasting with a bayesian mixed frequency model with stochastic volatility. Working paper. 
Mariano, R. and Y. Murasawa (2003). A new coincident index of business cycles based on monthly and quarterly series. Journal of Applied Econometrics 18, 427-443.

Mehra, R. (1970). On the identification of variances and adaptive kalman filtering. IEEE Transactions on Automatic Control 15(2).

Rao, R. R. (1962). Relations between Weak and Uniform Convergence of Measures with Applications. The Annals of Mathematical Statistics 33(2), 659-680.

Stock, J. H. and M. Watson (1990). A probability model of the coincident economic indicators. In K. Lahiri and G. H. Moore (Eds.), Leading Economic Indicators, pp. 63-89. Cambridge: Cambridge University Press.

Straumann, D. and T. Mikosch (2006). Quasi-maximum-likelihood estimation in conditionally heteroeskedastic time series: A stochastic recurrence equations approach. The Annals of Statistics 34(5), 2449-2495.

Trehan, B. (1989). "forecasting growth in current quarter real gnp. Federal Reserve Bank of S. Francisco Economic Review Winter, 39-52.

van der Vaart, A. W. (2000, June). Asymptotic Statistics (Cambridge Series in Statistical and Probabilistic Mathematics). Cambridge University Press.

White, H. (1994). Estimation, Inference and Specification Analysis. Cambridge Books. Cambridge University Press.

Wohlrabe, K. (2009). Forecasting with mixed-frequency time series models. Munich Dissertations in Economics 9681. 